\title{
STRUCTURE OF STABLE SOLUTIONS OF A ONE-DIMENSIONAL VARIATIONAL PROBLEM
}

\author{
NUNG KWAN YIP ${ }^{1}$
}

\begin{abstract}
We prove the periodicity of all $H^{2}$-local minimizers with low energy for a one-dimensional higher order variational problem. The results extend and complement an earlier work of Stefan Müller which concerns the structure of global minimizer. The energy functional studied in this work is motivated by the investigation of coherent solid phase transformations and the competition between the effects from regularization and formation of small scale structures. With a special choice of a bilinear double well potential function, we make use of explicit solution formulas to analyze the intricate interactions between the phase boundaries. Our analysis can provide insights for tackling the problem with general potential functions.
\end{abstract}

Mathematics Subject Classification. 47J20, 49K20, 34K26.

Received June 1, 2005. Revised October 19, 2005.

\section{InTRODUCTION AND STATEMENTS OF THEOREMS}

In this paper, we study the structure of $H^{2}$-local minimizers for the following functional:

$$
\mathcal{E}(u)=\int_{0}^{1} \epsilon^{2} u_{x x}^{2}+W\left(u_{x}\right)+u^{2} \mathrm{~d} x \quad \text { subject to } u_{x}(0)=u_{x}(1)=0
$$

where $W$ is some double-well potential function usually taken to be $W(p)=\left(p^{2}-1\right)^{2}$. In order to facilitate the use of explicit solution formulas, in the present paper, we consider the special form of $W(p)=(|p|-1)^{2}$ so that

$$
W^{\prime}(p)=\left\{\begin{array}{l}
2(p-1), p>0 \\
2(p+1), p<0
\end{array}, \quad W^{\prime}\left(0^{+}\right)-W^{\prime}\left(0^{-}\right)=-4 \quad \text { and } \quad W^{\prime \prime}(p)=2-4 \delta_{0}(p) .\right.
$$

The reason for this choice is that the corresponding Euler-Lagrange equation for (1) is given by a linear differential equation with constant coefficients together with some linear jump conditions for the solutions.

As our goal is to investigate the relation between the critical points of $\mathcal{E}$ and periodic patterns, we first present the solution of a unit cell problem. For each $l>0$, let $P(x, l)$ be the function which solves the following

Keywords and phrases. Higher order functional, local minimizer.

1 Department of Mathematics, Purdue University, USA; yip@math.purdue.edu 
boundary value problem:

$$
\left\{\begin{array}{c}
\epsilon^{2} P_{x x x x}(x, l)=P_{x x}(x, l)-P(x, l) \quad \text { and } \quad P_{x}(x, l)>0 \quad \text { for } x \in\left(-\frac{l}{2}, \frac{l}{2}\right), \\
P_{x}\left(-\frac{l}{2}, l\right)=P_{x}\left(\frac{l}{2}, l\right)=0 \\
\epsilon^{2} P_{x x x}\left(-\frac{l}{2}, l\right)=\epsilon^{2} P_{x x x}\left(\frac{l}{2}, l\right)=-1
\end{array}\right.
$$

(existence and uniqueness of which will be stated in Prop. 3.3). Now let $N=\frac{1}{l}$ be an integer and $Q^{N}(x)$ be the following periodic version of $P(\cdot, l)$ :

$$
Q^{N}(i l+y)=P\left((-1)^{i}\left(y-\frac{l}{2}\right), l\right) \quad \text { for } \quad 0 \leq y \leq l \quad \text { and } \quad i=0,1,2 \ldots N-1 .
$$

The following are our main results.

Theorem 1.1. There exist constants $C_{L}<10, l_{*}$ and $\epsilon_{*}>0$ such that for any $0<\epsilon<\epsilon_{*}$, if $C_{L} \epsilon|\ln \epsilon|<l<l_{*}$ and $N=\frac{1}{l}$ is an integer, then the function $\pm Q^{N}(x)$ defined as in (3) is a stable stationary point of the functional $\mathcal{E}(\cdot)$.

Theorem 1.2. There exist constants $C_{E}>\frac{3}{200}$ and $\epsilon_{*}>0$ such that for any $0<\epsilon<\epsilon_{*}$, if $u$ is a weakly stable stationary point of $\mathcal{E}(\cdot)$ satisfying

$$
\mathcal{E}(u) \leq \frac{C_{E}}{|\ln \epsilon|},
$$

then $u(\cdot)=Q^{N}(\cdot)$ or $-Q^{N}(\cdot)$ for some positive integer $N$.

Theorem 1.3. There exist constants $C_{S}<10$ and $\epsilon_{*}>0$ such that for all $0<\epsilon<\epsilon_{*}$ and $0<C<C_{S}$, if $l=C \epsilon|\ln \epsilon|$ and $N=\frac{1}{l}$ is an integer, then $Q^{N}(\cdot)$ is unstable.

(Note that for the $l$ in this theorem, $\mathcal{E}\left(Q^{N}(\cdot)\right)=O\left(\frac{1}{\ln \epsilon}\right)$.)

The notions of stationary points, their stability and relationship to local minimizers of $\mathcal{E}$ will be given in Section 2. All of the above results can be extended in a natural way to the Dirichlet $\{u(0)=u(1)=0\}$ and periodic $\left\{u(0)=u(1) ; u_{x}(0)=u_{x}(1)\right\}$ boundary conditions.

Our results in essence establish the fact that if $u$ is a stationary point of $\mathcal{E}(\cdot)$ of low enough energy, then it is stable if and only if it is periodic. Theorem 1.3 states that we have captured the correct range of the energy values in terms of the stability properties of periodic structures. Our work hence extends and complements the following theorem of S. Müller which studies the structure of global minimizer for the functional (1) in the case of $W(p)=\left(p^{2}-1\right)^{2}$. Let $A_{0}=2 \int_{-1}^{1} W^{\frac{1}{2}}(p) \mathrm{d} p$.

Theorem 1.4 [12]. There exists an $\epsilon_{*}>0$ such that for $0<\epsilon<\epsilon_{*}$, if $u$ is a global minimizer of $\mathcal{E}(\cdot)$ in the class of periodic functions:

$$
H_{\#}^{2}(0,1)=\left\{u \in H^{2}(0,1): u(0)=u(1) \text { and } u_{x}(0)=u_{x}(1)\right\},
$$

then $u$ is periodic with minimal period $T^{\epsilon}=2\left(6 A_{0} \epsilon\right)^{\frac{1}{3}}+O\left(\epsilon^{\frac{2}{3}}\right)$. Moreover,

$$
u\left(x+\frac{T^{\epsilon}}{2}\right)=-u(x) \text { for } x \in(0,1) \text { and } \mathcal{E}(u)=\frac{1}{4}\left(6 A_{0} \epsilon\right)^{\frac{2}{3}}+O\left(\epsilon^{\frac{4}{3}}\right) .
$$

The motivations for the investigation of (1) are twofold. One comes from the study of coherent solid-solid transformations which very often give rise to some fine scale mixtures of different phases with characteristic length scales. The formulation of energy minimization in the modeling of these transformations can be found in $[3,4,8]$. In the one-dimensional setting, the phenomenology of the formation of mixtures of different phases can be captured to sufficient degree by (1) and related functionals. It is the combination of the strain energy $W\left(u_{x}\right)$ 
which favors $u_{x}$ to be 1 or -1 and the elastic foundation term $u^{2}$ that leads to the formation of fine scale structures. However, definite length scales are determined by the incorporation of the strain gradient or surface energy term $\epsilon^{2} u_{x x}^{2}$. We refer to [16] for a more detail account of (1). The works [13-15] also consider some nonlocal versions of $u^{2}$. A two-dimensional model can be found in $[9,10]$.

The second motivation is that (1) can be viewed as a regularization of some functional which is not lowersemicontinuous. For example, the following functional

$$
\mathcal{E}_{0}(u)=\int_{0}^{1}\left(W\left(u_{x}\right)+u^{2}\right) \mathrm{d} x
$$

does not have a minimizer. Instead it possesses a minimizing sequence $\left\{u^{\delta}\right\}_{\delta>0}$ of the form that $u_{x}^{\delta}$ oscillates between 1 and -1 with increasing frequencies such that $\left\|u^{\delta}\right\|_{\infty} \rightarrow 0$. The incorporation of the higher order term $\epsilon^{2} u_{x x}^{2}$ in (5) penalizes the oscillations in $u_{x}$ and hence limits their number. By simple compactness arguments, a minimizer for (1) exists. Furthermore, formal reasoning leads to that (1) for $0<\epsilon \ll 1$ can be approximated by

$$
\mathcal{E}_{*}^{1}(u)=\int_{0}^{1} \frac{1}{2} \epsilon A_{0}\left|u_{x x}\right|+u^{2} \mathrm{~d} x \quad \text { subject to }\left|u_{x}\right|=1 \text { a.e. }
$$

where $A_{0}=2 \int_{-1}^{1} \sqrt{W(p)} \mathrm{d} p$, or even more explicitly, by

$$
\mathcal{E}_{*}^{2}(u)=\int_{0}^{1} u^{2} \mathrm{~d} x+\epsilon A_{0} \times\left(\text { number of times } u_{x} \text { changes between } 1 \text { and }-1\right)
$$

with $u$ subjected to the same constraint. Assuming the validity of such an approximation, the minimizer $u$ of (1) can then be approximated by the minimizer of $\mathcal{E}_{*}^{1}$ or $\mathcal{E}_{*}^{2}$. From this we infer that there is a collection of roughly equidistant points $\left\{c_{i}\right\}$ 's at which $u_{x}$ changes between 1 and -1 . In between these points, $\left|u_{x}\right|$ stays very close to 1 so that $u$ is very much like a sawtooth function. We call the transition of $u_{x}$ between 1 and -1 an interface and the region between any two interfaces a phase segment or simply a segment. See the following Figure 1.
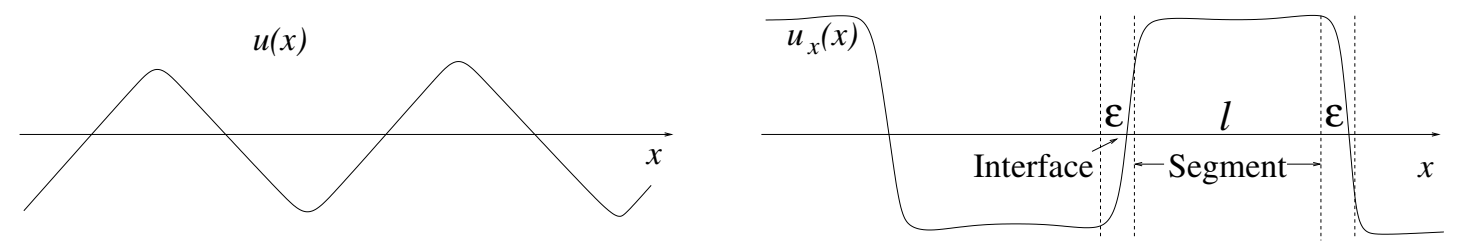

Figure 1. A typical pattern of $u(x)$ and its derivative $u_{x}(x)$.

Another interesting feature of the functional (1) is the competition between multiple - two - length scales. One is $\epsilon$, the interfacial width and the other is $l$, the length of the segment. The result of this competition is that $l=O\left(\epsilon^{\frac{1}{3}}\right)$ for the global minimizer of (1). Rigorous justification of the relationship between $\mathcal{E}, \mathcal{E}_{*}^{1}$ and $\mathcal{E}_{*}^{2}$ falls in the regime of $\Gamma$-convergence of functionals. We refer to $[2,12]$ for a discussion of this approach for the present problem. [14] also uses this method to study (1) but with $u^{2}$ replaced by $\epsilon u^{2}$ in which case, $l=O(1)$ and hence there is only one small length scale left in the problem.

In order to study dynamic problems such as gradient flows, the classification of critical points is also important as the ultimate observed patterns of the phase transformations are consequences of both energetic and kinetic effects. Our results state that (1) has many critical points but those having low enough energy and stability property are in fact periodic. Hence a time dependent solution can very likely fall into the basins of attraction of these local minimizers and stay there indefinitely. We refer to [1] for some experimental investigations of such phenomena which is relevant to the current functional. 
There are relatively few methods that can be used to describe the structure of critical points compared with those that can prove the existence of them. In addition, as (1) is a higher order variational problem, the usual techniques for second order equations are not easily at our disposal. Even though it is plausible to use the approach of $\Gamma$-convergence to deduce that there exist local-minimizers which are nearly periodic (see [11] for a general framework), to conclude other statements such as the existence and characterization of other types of local minimizers or critical points, more precise estimates are needed.

Our approach, originated in [12], resembles that of asymptotic expansion. Theorem 1.1 is proved by following quite closely the approach of [12], Theorem 5.1. However, the proof of Theorem 1.2 is much more delicate. It requires careful analysis of the interactions between the interfaces. Our method can potentially be used to find all critical points which do not fall easily in the regime of $\Gamma$-convergence.

Our analysis is made possible by the special choice of $W$. It allows the use of explicit solution formulas. Such a choice is also used in $[16,17]$ in which detailed analysis is performed for the case when the number of interfaces is small. We believe that our results can provide useful insights to tackle the case of general $W$ 's in terms of what types of quantities and estimates to be looked upon.

It is instructive to compare (1) with the following Allen-Cahn functional:

$$
\mathcal{F}(v)=\int_{0}^{1} \epsilon^{2} v_{x}^{2}+W(v) \mathrm{d} x \quad \text { subject to } v_{x}(0)=v_{x}(1)=0 \text { and } \int_{0}^{1} v \mathrm{~d} x=0
$$

for which all the critical points are periodic and unstable except the global minimizer which has only one interface where $v$ changes between 1 and -1 [6]. This model thus cannot capture any fine scale structures. Furthermore, the time dependent problem for the above functional demonstrates the existence of metastable states - interfaces move with exponentially small speeds [5,7]. This is due to the fact that each pair of adjacent interfaces gives rise to an exponentially small eigenvalue. However, for (1), we are dealing with algebraically small eigenvalue.

\subsection{Outline of Proof}

Using the analysis of [12], we can easily deduce that any critical point $u$ of $\mathcal{E}$ with low enough energy has a sawtooth shape so that $\left|u_{x}\right| \approx 1$ away from the zeros of $u_{x}$ or the interfacial regions. This is seen by considering the Euler-Lagrange equation of (1) which for smooth $W$ reads:

$$
\epsilon^{2} u_{x x x x}=\frac{W^{\prime \prime}\left(u_{x}\right)}{2} u_{x x}-u \text {. }
$$

If the distance between the interfaces are long, then the behavior of $u$ can be described by $\epsilon^{2} u_{x x x x}=\frac{W^{\prime \prime}\left(u_{x}\right)}{2} u_{x x}$ for $x$ near the interface and by $\frac{W^{\prime \prime}\left(u_{x}\right)}{2} u_{x x}=u$ for $x$ far away from the interface. From these observations, sharp estimates can be deduced. Theorem 1.1 is proved by showing that the minimum energy $E(l)$ of a monotonic function $u$ over a segment of length $l$ is a convex function of $l$. This is very similar to the approach of [12], Theorem 5.1.
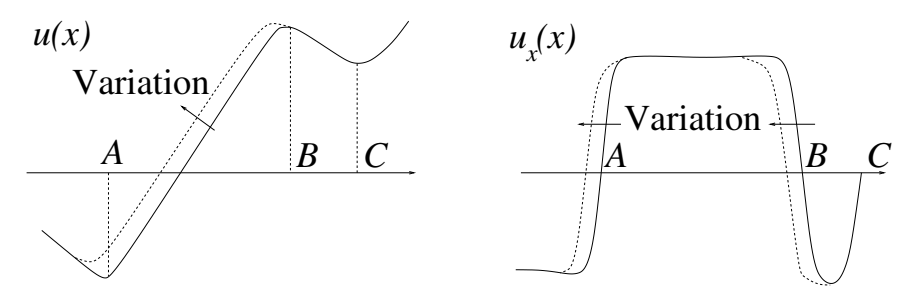

Figure 2. An example of a variation for a pattern with a short segment adjacent to a long segment. 
However, in order to deduce the periodicity of stable patterns, we need to consider the possibility of short segments. In this case, the above separation of scales is not quite useful. We need much more precise information about the interactions between the interfaces. The core idea of this paper is to capture these interactions by means of a propagation map which relates the behaviors of $u$ at both ends of an segment. With this map, we can find out the relationship between the lengths of adjacent segments.

As an illustrative example, consider the configuration of $u$ and $u_{x}$ as in Figure 2. In this example, the lengths of $|A B|$ and $|B C|$ differ substantially from each other. We now vary $u$ by moving the interfaces at $A$ and $B$. The second variation of $\mathcal{E}$ at $u$ is found to be:

$$
\partial^{2} \mathcal{E}(u) \approx-\frac{O\left(\mathrm{e}^{-\frac{|B C|}{\epsilon}}\right)}{\epsilon}+|A B| .
$$

In order to determine the sign of the second variation, we need to analyze the relationship between $|B C|$ and $|A B|$. This procedure falls into an unfortunately large number of cases which we will check one by one. From this analysis, we conclude that each short segment leads to an unstable eigen-mode and hence in order for $u$ to be a stable critical point, it can only have long segments. Then a reasoning using first variation shows that $u$ must be nearly periodic. A final step using an implicit function theorem type argument concludes the periodicity of $u$.

The outline of the paper is as follows. Section 2 gives the definitions of critical points of $\mathcal{E}$, their (in-)stability, and some preliminary estimates. Section 3 introduces the propagation map which will be used throughout the paper. The proofs of the main theorems will be given in Sections 4-6. The Appendix states some regularity property of the critical points of $\mathcal{E}$ and also provides some explicit examples of unstable solutions.

\section{EULER-LAGRANGE EQUATION}

The Euler-Lagrange equation for the functional $\mathcal{E}$ is formally given by

$$
\epsilon^{2} u_{x x x x}=\frac{W^{\prime \prime}\left(u_{x}\right)}{2} u_{x x}-u
$$

or, in the integrated form:

$$
\epsilon^{2} u_{x x x}=\frac{W^{\prime}\left(u_{x}\right)}{2}-\int_{0}^{x} u(y) \mathrm{d} y+C \text { for some constant } C .
$$

Note that there is an ambiguity about the meaning of $W^{\prime}(0)$ which occurs at the points where $u_{x}=0$. From Theorem A.2, it is sufficient to consider those solutions $u$ with only a finite number of zeros for $u_{x}$ so that $u$ is piecewise monotone. In this way, the ambiguity of $W^{\prime}(0)$ can be handled by imposing appropriate jump conditions for $u$. However, we do not a priori limit the number of the zeros. The necessary formulation for the solutions of (9) will be given next.

Definition 2.1. A function $u$ is said to belong to class $\mathcal{Z}$ if $u \in C^{2}([0,1])$ and $[0,1]$ can be partitioned into a finite number of segments $\left\{\left[c_{i}, c_{i+1}\right]: i=0,1, \ldots N-1\right\}$ for some positive integer $N$ and $c_{i}$ 's: $0=c_{0}<c_{1}<$ $\cdots<c_{N-1}<c_{N}=1$ such that

1. $u$ is monotone $\left(u_{x} \geq 0\right.$ or $\left.u_{x} \leq 0\right)$ in each of the segment $\left(c_{i}, c_{i+1}\right)$ and the sign of $u_{x}$ alternates between adjacent segments, i.e. $u_{x}$ changes sign across the $c_{i}$ 's;

2. the zeros of $u_{x}$ are isolated. In particular, $u_{x}$ is not identically zero in any interval.

Remark. A priori, there can be two kinds of zeros for $u_{x}$. One is the sign-changing zero $c_{i}$ which indicates the location of the interface. The other is the interior zero which lies in between any two adjacent $c_{i}$ 's. The sign of $u_{x}$ does not change across these zeros. We are mainly concerned with the sign-changing zeros while the interior zeros can be shown not to occur for local minimizers or any stationary point of $\mathcal{E}$ with low energy. 
In the following, the notation $[f](x)$ refers to $f\left(x^{+}\right)-f\left(x^{-}\right)$.

Definition 2.2. A function $u \in \mathcal{Z}$ is called a solution of (9) if the following hold for all $i$ :

$$
\begin{gathered}
\epsilon^{2} u_{x x x x}=u_{x x}-u, x \in\left(c_{i}, c_{i+1}\right) \\
u_{x}\left(c_{i}\right)=0 \text { and } u_{x} \geq 0\left(\text { or } u_{x} \leq 0\right) \quad \text { for all } x \in\left(c_{i}, c_{i+1}\right) \\
{\left[\epsilon^{2} u_{x x x}\right]\left(c_{i}\right)\left(=\left[\frac{1}{2} W^{\prime}\left(u_{x}\right)\right]\left(c_{i}\right)\right)=-2 \operatorname{sgn}^{*}\left(u_{x x}\left(c_{i}\right)\right) .} \\
u_{x}(0)=u_{x}(1)=0, \quad \epsilon^{2} u_{x x x}(0)=-\operatorname{sgn}^{*}\left(u_{x x}(0)\right), \quad \epsilon^{2} u_{x x x}(1)=\operatorname{sgn}^{*}\left(u_{x x}(1)\right) .
\end{gathered}
$$

In (13), the symbol $2 \operatorname{sgn}^{*}\left(u_{x x}\left(c_{i}\right)\right)$ refers to $\operatorname{sgn}\left(u_{x}\left(c_{i}^{+}\right)\right)-\operatorname{sgn}\left(u_{x}\left(c_{i}^{-}\right)\right)$. In $(14), \operatorname{sgn}^{*}\left(u_{x x}(0)\right)$ and $\operatorname{sgn}^{*}\left(u_{x x}(1)\right)$ refer to $\operatorname{sgn}\left(u_{x}\left(0^{+}\right)\right)$and $-\operatorname{sgn}\left(u_{x}\left(1^{-}\right)\right)$respectively.

Remark. Since $u$ satisfies (11) in $\left(c_{i}, c_{i+1}\right)$, it is analytic. In addition, as $u_{x}$ is assumed not to be identically zero, the zeros of $u_{x}$ inside each segment are automatically isolated and also do not cluster at the $c_{i}$ 's. Hence the quantities $\operatorname{sgn}\left(u_{x}\left(c_{i}^{ \pm}\right)\right)$'s are well defined.

In fact, the following proposition states that we can replace the sgn* by the usual sgn.

Proposition 2.3. If $u \in \mathcal{Z}$ and satisfies (13) and (14), then $u_{x x}\left(c_{i}\right) \neq 0$ for $i=0,1, \ldots N$. Hence $\operatorname{sgn}^{*}\left(u_{x x}\left(c_{i}\right)\right)=\operatorname{sgn}\left(u_{x x}\left(c_{i}\right)\right)$.

Proof. The proof follows easily by contradiction. Suppose $u_{x x}\left(c_{i}\right)=0$ and $u_{x}\left(c_{i}^{+}\right)>0$ and $u_{x}\left(c_{i}^{-}\right)<0$. Then we have $u_{x x x}\left(c_{i}^{+}\right) \geq 0$ and $u_{x x x}\left(c_{i}^{-}\right) \leq 0$ which contradicts (13). The other cases follow similarly.

The following proposition motivates our definitions of solutions and stability.

Proposition 2.4. Let $u \in \mathcal{Z}$ and $\varphi \in \mathcal{V}^{\infty}=C^{\infty}([0,1]) \bigcap\left\{\varphi_{x}(0)=\varphi_{x}(1)=0\right\}$. Then the following statements hold.

1. The first variation of $\mathcal{E}(u)$ with respect to $\varphi$, defined as $\left.\frac{1}{2} \frac{\mathrm{d}}{\mathrm{d} t} \mathcal{E}(u+t \varphi)\right|_{t=0}$ equals:

$$
\begin{aligned}
\sum_{i=1}^{N-1}\left\{\left[\epsilon^{2} u_{x x x}\right]\left(c_{i}\right)+2 \operatorname{sgn}^{*}\left(u_{x x}\left(c_{i}\right)\right)\right\} \varphi\left(c_{i}\right)+\left\{\epsilon^{2} u_{x x x}(0)+\operatorname{sgn}^{*}\left(u_{x x}(0)\right)\right\} \varphi(0) \\
-\left\{\epsilon^{2} u_{x x x}(1)-\operatorname{sgn}^{*}\left(u_{x x}(1)\right)\right\} \varphi(1)+\sum_{i=0}^{N-1} \int_{c_{i}(t)}^{c_{i+1}(t)}\left(\epsilon^{2} u_{x x x x}-u_{x x}+u\right) \varphi \mathrm{d} x
\end{aligned}
$$

In particular, $u \in \mathcal{Z}$ is a solution (in the sense of Def. 2.2) if and only if $\left.\frac{\mathrm{d}}{\mathrm{d} t} \mathcal{E}(u+t \varphi)\right|_{t=0}=0$ for all $\varphi \in \mathcal{V}^{\infty}$.

2. Suppose $u_{x x}\left(c_{i}\right) \neq 0$ (which holds when $u$ is a solution). Then there are $C^{1}$ functions $c_{i}(t)$ such that $c_{i}(0)=c_{i}$, and for small $t, u_{x}\left(c_{i}(t)\right)+t \varphi_{x}\left(c_{i}(t)\right)=0$. Furthermore,

$$
\dot{c}_{i}(0)=-\frac{\varphi_{x}\left(c_{i}\right)}{u_{x x}\left(c_{i}\right)} .
$$

(In essence, the $c_{i}(t)$ 's are the sign-changing zeros of $u_{x}+t \varphi_{x}$. )

3. Suppose $u_{x x}\left(c_{i}\right) \neq 0$ (which holds when $u$ is a solution) and $u_{x} \neq 0$ for $x \in\left(c_{i}, c_{i+1}\right)$, i.e. $u_{x}$ has no interior zeros, then the second variation of $\mathcal{E}(u)$ with respect to $\varphi$, defined as $\left.\frac{1}{2} \mathrm{~d}^{2} \mathcal{d} t^{2} \mathcal{E}(u+t \varphi)\right|_{t=0}$ equals:

$$
\int_{0}^{1} \epsilon^{2} \varphi_{x x}^{2}+\varphi_{x}^{2}+\varphi^{2} \mathrm{~d} x-2 \sum_{i=0}^{N-1} \frac{\varphi_{x}^{2}\left(c_{i}\right)}{\left|u_{x x}\left(c_{i}\right)\right|}
$$


Remark. In general, if $u_{x}$ has interior zeros, $\partial^{2} \mathcal{E}(u, \varphi) \neq\left.\frac{1}{2} \frac{\mathrm{d}^{2}}{\mathrm{~d} t^{2}} \mathcal{E}(u+t \varphi)\right|_{t=0}$. But by Theorem A.2(3) and estimate (32) in Proposition 2.7 (presented later), this will not be an issue in our work. Again, if $u$ is a solution, the fact that $u_{x x}\left(c_{i}\right) \neq 0$ follows from Proposition 2.3.

Proof of (15). To prove (15), we compute

$$
\begin{aligned}
\left.\frac{1}{2} \frac{\mathrm{d}}{\mathrm{d} t} \mathcal{E}(u+t \varphi)\right|_{t=0} & =\frac{1}{2} \frac{\mathrm{d}}{\mathrm{d} t} \int_{0}^{1} \epsilon^{2}\left(u_{x x}+t \varphi_{x x}\right)^{2}+\left(\left|u_{x}+t \varphi_{x}\right|-1\right)^{2}+\left.(u+t \varphi)^{2} \mathrm{~d} x\right|_{t=0} \\
& =\int_{0}^{1} \epsilon^{2} u_{x x} \varphi_{x x}+u_{x} \varphi_{x}+u \varphi \mathrm{d} x-\lim _{t \rightarrow 0} \int_{0}^{1} \frac{\left|u_{x}+t \varphi_{x}\right|-\left|u_{x}\right|}{t} \mathrm{~d} x .
\end{aligned}
$$

The last term of the above equals

$$
\lim _{t \rightarrow 0} \int_{\left\{u_{x} \neq 0\right\}} \frac{\left|u_{x}+t \varphi_{x}\right|-\left|u_{x}\right|}{t} \mathrm{~d} x+\lim _{t \rightarrow 0} \int_{\left\{u_{x}=0\right\}} \frac{\left|t \varphi_{x}\right|}{t} \mathrm{~d} x .
$$

As $u_{x}$ is assumed to have isolated zeros, we can ignore the second integral of (18). To simplify the first integral, note that

$$
\left|\frac{|a+t b|-|a|}{t}\right| \leq|b| \text { for all } a, b, t \text { and } \lim _{t \rightarrow 0} \frac{|a+t b|-|a|}{t}=\operatorname{sgn}(a) b \text { if } a \neq 0 .
$$

By the Lebesgue Dominated Convergence Theorem, we have

$$
\lim _{t \rightarrow 0} \int_{\left\{u_{x} \neq 0\right\}} \frac{\left|u_{x}+t \varphi_{x}\right|-\left|u_{x}\right|}{t} \mathrm{~d} x=\int_{0}^{1} \operatorname{sgn}\left(u_{x}\right) \varphi_{x} \mathrm{~d} x
$$

The above steps thus lead to

$$
\left.\frac{1}{2} \frac{\mathrm{d}}{\mathrm{d} t} \mathcal{E}(u+t \varphi)\right|_{t=0}=\int_{0}^{1} \epsilon^{2} u_{x x} \varphi_{x x}+\left(\left|u_{x}\right|-1\right) \operatorname{sgn}\left(u_{x}\right) \varphi_{x}+u \varphi \mathrm{d} x .
$$

Performing integration by parts twice on each segment $\left(c_{i}, c_{i+1}\right)$ and using the facts that $[u+t \varphi]=\left[u_{x}+t \varphi_{x}\right]=$ $\left[u_{x x}+t \varphi_{x x}\right]=0$ at $x=c_{i},(15)$ follows.

It is then easy to infer the equivalence of $u$ being a solution in the sense of Definition 2.2 and the vanishing of its first variation for all $\varphi \in \mathcal{V}^{\infty}$.

Proof of (16). It follows from elementary computations. Since $u_{x}$ and $\varphi_{x}$ are both $C^{1}$ functions and $u_{x x}\left(c_{i}\right) \neq 0$, we can use the implicit function to show the existence of $C^{1}$ functions $c_{i}(t)$ (for small enough $t$ ) satisfying $u_{x}\left(c_{i}(t)\right)+t \varphi_{x}\left(c_{i}(t)\right)=0$. Furthermore,

$$
u_{x x}\left(c_{i}(t)\right) \dot{c}_{i}(t)+t \varphi_{x x}\left(c_{i}(t)\right) \dot{c}_{i}(t)+\varphi_{x}\left(c_{i}(t)\right)=0
$$

which leads to (16).

Proof of (17). We start from the first variation, $\frac{1}{2} \frac{\mathrm{d}}{\mathrm{d} t} \mathcal{E}(u+t \varphi)$ which equals

$$
\begin{aligned}
\frac{1}{2} \frac{\mathrm{d}}{\mathrm{d} t} \sum_{i=0}^{N-1} \int_{c_{i}(t)}^{c_{i+1}(t)} \epsilon^{2}\left(u_{x x}\right. & \left.+t \varphi_{x x}\right)^{2}+\left(u_{x}+t \varphi_{x}-\operatorname{sgn}\left(u_{x}+t \varphi_{x}\right)\right)^{2}+(u+t \varphi)^{2} \mathrm{~d} x \\
& =\sum_{i=0}^{N-1} \int_{c_{i}(t)}^{c_{i+1}(t)} \epsilon^{2}\left(u_{x x}+t \varphi_{x x}\right) \varphi_{x x}+\left(u_{x}+t \varphi_{x}-\operatorname{sgn}\left(u_{x}+t \varphi_{x}\right)\right) \varphi_{x}+(u+t \varphi) \varphi \mathrm{d} x
\end{aligned}
$$


The condition of no interior zeros for $u_{x}$ is to ensure that for $t$ small enough, there is no new zeros for $u_{x}+t \varphi_{x}$ other than the sign-changing ones, $c_{i}(t)$ 's. Now (17) follows by differentiating $(21)$ :

$$
\frac{1}{2} \frac{\mathrm{d}^{2}}{\mathrm{~d} t^{2}} \mathcal{E}(u+t \varphi)=\sum_{i=0}^{N-1} 2 \operatorname{sgn}^{*}\left(u_{x x}\left(c_{i}(t)\right)\right) \varphi_{x}\left(c_{i}(t)\right) \dot{c}_{i}(t)+\int_{c_{i}(t)}^{c_{i+1}(t)} \epsilon^{2} \varphi_{x x}^{2}+\varphi_{x}^{2}+\varphi^{2} \mathrm{~d} x
$$

and then utilizing (16).

With the above definition of solutions, we have the next two useful formula:

1. the integrated version of (11): for $x \in\left(c_{i}, c_{i+1}\right)$,

$$
\epsilon^{2} u_{x x x}(x)-\epsilon^{2} u_{x x x}\left(c_{i}^{+}\right)=u_{x}(x)-\int_{c_{i}}^{x} u(y) \mathrm{d} y
$$

2. multiplying the previous equation by $u_{x x}$ and integrating from $c_{i}^{+}$to $x$ gives:

$\epsilon^{2} u_{x x}^{2}(x)-\left(u_{x}(x)-\operatorname{sgn}\left(u_{x}\right)\right)^{2}=\epsilon^{2} u_{x x}^{2}\left(c_{i}\right)-1+2\left(\epsilon^{2} u_{x x x}\left(c_{i}^{+}\right)+\operatorname{sgn}\left(u_{x}\right)\right) u_{x}-2\left(\int_{c_{i}}^{x} u(y) \mathrm{d} y\right) u_{x}(x)+u^{2}(x)-u^{2}\left(c_{i}\right)$.

We are now ready to introduce the notion of stability and instability used in the statements of our main theorems. For all $u \in \mathcal{Z}$, such that $u_{x x}\left(c_{i}\right) \neq 0$, we make the following definitions:

$$
\begin{gathered}
\mathcal{V}=H^{2}(0,1) \cap\left\{\varphi: \varphi_{x}(0)=\varphi_{x}(1)=0\right\} \\
\mathcal{D}(u, \varphi)=\int_{0}^{1} \epsilon^{2} \varphi_{x x}^{2}+\varphi_{x}^{2} \mathrm{~d} x-2 \sum_{i=0}^{N-1} \frac{\varphi_{x}^{2}\left(c_{i}\right)}{\left|u_{x x}\left(c_{i}\right)\right|} \text { for } u \in \mathcal{Z} \text { and } \varphi \in \mathcal{V} \\
\text { and } \partial^{2} \mathcal{E}(u, \varphi)=\mathcal{D}(u, \varphi)+\int_{0}^{1} \varphi^{2} \mathrm{~d} x
\end{gathered}
$$

Definition 2.5. A function $u \in \mathcal{V}$ is called a stationary point of $\mathcal{E}$ if for all $\varphi \in \mathcal{V}$,

$$
\left.\frac{\mathrm{d}}{\mathrm{d} t} \mathcal{E}(u+t \varphi)\right|_{t=0} \text { exists and equals to } 0 \text {. }
$$

Definition 2.6. A solution $u \in \mathcal{Z}$ of (9) is called (weakly) stable if

$$
\text { for all } \varphi \neq 0, \in \mathcal{V}, \partial^{2} \mathcal{E}(u, \varphi)(\geq)>0 \text {. }
$$

It is called unstable if

$$
\text { there exists a } \varphi \in \mathcal{V} \text { such that } \partial^{2} \mathcal{E}(u, \varphi)<0 \text {. }
$$

Remark. This paper will only consider the critical points of $\mathcal{E}$ in the function space $\mathcal{Z}$ and their stability properties. The reason why this is sufficient will be explained by the regularity results in Theorem A.2 which also gives the relation between the above definition of stability and the notion of $H^{2}$-local minimizers.

Next, we prove some preliminary estimates for any stationary point of the functional (1) having small enough energy. The results are crude but important to prepare for our proof later. They are similar to the results of [12], Section 2, and the proof will hence be omitted (or see [18]).

Proposition 2.7. Let $u \in \mathcal{Z}$ be a solution of (9) and $E$ be the energy of $u$ :

$$
E=\mathcal{E}(u)=\int_{0}^{1} \epsilon^{2} u_{x x}^{2}+W\left(u_{x}\right)+u^{2} \mathrm{~d} x
$$


Then there exist constants $E_{*}<1, \epsilon_{*}, C_{1}, C_{2}>0$ such that for all $\epsilon<\epsilon_{*}$ and $u$ with $E<E_{*}$, the following statements hold for all $i=0,1, \ldots N-1$ and $x \in[0,1]$ :

$$
\begin{gathered}
\left|\int_{a}^{b} u(y) d y\right| \leq C_{1} E^{\frac{1}{2}} ; \\
|| \epsilon^{2} u_{x x x}\left(c_{i}^{ \pm}\right)|-1| \leq C_{1} E^{\frac{1}{2}} ; \\
\left|u_{x}(x)\right| \leq 1+C_{1} E^{\frac{1}{2}} ; \\
|u(x)| \leq C_{1} E^{\frac{1}{3}} ; \\
\left|\epsilon^{2} u_{x x}^{2}\left(c_{i}\right)-1\right| \leq C_{1} E^{\frac{1}{2}} ; \\
|| u_{x}(\bar{x})|-1| \leq C_{1} E^{\frac{1}{4}} \quad \text { at all } \bar{x} \text { such that } u_{x x}(\bar{x})=0 ; \\
C_{2} \epsilon|\ln E| \leq\left(c_{i+1}-c_{i}\right) \leq C_{1} E^{\frac{1}{3}} .
\end{gathered}
$$

In particular, if $E$ is small enough, (32) states that within any segment $\left(c_{i}, c_{i+1}\right), u_{x}$ does not have any interior zero and also there must be a point $\bar{x}$ such that $\left|u_{x}(\bar{x})\right|>\frac{1}{2}$. Furthermore, (33) states that the length of any segment cannot be too long but is much longer than $\epsilon$.

As an application of the above estimates, we have the following lower bound for the energy inside any segment $\left(c_{i}, c_{i+1}\right)$ :

Lemma 2.8. Under the same hypothesis as Proposition 2.7, then for all $\epsilon<\epsilon_{*}$ and $u$ such that $E \leq E_{*}$, it holds that

$$
\int_{c_{i}}^{c_{i+1}} \epsilon^{2} u_{x x}^{2}+W\left(u_{x}\right) \mathrm{d} x \geq \frac{3}{4} \epsilon \text { for all } i .
$$

Proof.

$$
\int_{c_{i}}^{c_{i+1}} \epsilon^{2} u_{x x}^{2}+W\left(u_{x}\right) \mathrm{d} x \geq \epsilon \int_{c_{i}}^{c_{i+1}} \epsilon u_{x x}^{2}+\frac{1}{\epsilon} W\left(u_{x}\right) \mathrm{d} x \geq 2 \epsilon \int_{0}^{\frac{1}{2}} \sqrt{W\left(u_{x}\right)}\left|u_{x x}\right| \mathrm{d} x=\frac{3}{4} \epsilon
$$

since from (32), there must be a point $\bar{x} \in\left(c_{i}, c_{i+1}\right)$ such that $\left|u_{x}(\bar{x})\right| \geq \frac{1}{2}$.

\section{Propagation map for Euler-Lagrange equation}

Here we introduce the propagation map which relates the boundary values at the end points of a segment over which the solution $u$ is monotone. Precisely, we consider the following boundary value problem:

$$
\epsilon^{2} u_{x x x x}-u_{x x}+u=0 \text { such that } u_{x}(0)=u_{x}(l)=0 \text { and } u_{x} \neq 0 \text { for } 0 \leq x \leq l .
$$

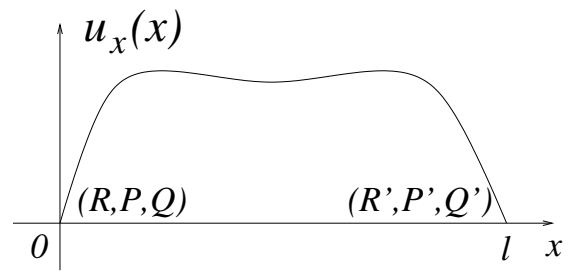

Figure 3. The boundary value problem for the propagation map.
For the case $u_{x}>0$, the initial and final values are denoted by:

$$
\begin{gathered}
u(0)=R, \quad \epsilon u_{x x}(0)=P, \quad \epsilon^{2} u_{x x x}(0)=-Q \\
u(l)=R^{\prime}, \quad \epsilon u_{x x}(l)=-P^{\prime}, \quad \epsilon^{2} u_{x x x}(l)=-Q^{\prime} .
\end{gathered}
$$

(See Fig. 3 on the left.) 
The existence of a unique solution to the above problem is proved in Proposition 3.3. In the actual application of the result, the segment $(0, l)$ will be one of the $\left(c_{i}, c_{i+1}\right)$ 's. As we are interested in the case of $\epsilon \rightarrow 0$ and $\mathcal{E}(u) \ll 1$, by Proposition 2.7, we only need to consider the regime of $l, R, R^{\prime}=o(1)$ and $P, P^{\prime}, Q, Q^{\prime}=1+o(1)$.

Our goal is to compute the following propagation map as a function of $l$ and $\epsilon$ :

$$
(R, P, Q) \longrightarrow_{l, \epsilon}\left(R^{\prime}, P^{\prime}, Q^{\prime}\right)
$$

This requires solving (34). Its characteristic polynomial is $\epsilon^{2} r^{4}-r^{2}+1=0$ which has four roots $\pm \Lambda$ and $\pm \lambda$ :

$$
\Lambda=\sqrt{\frac{1+\sqrt{1-4 \epsilon^{2}}}{2 \epsilon^{2}}}=\frac{1-\frac{1}{2} \epsilon^{2}+O\left(\epsilon^{4}\right)}{\epsilon} \quad \text { and } \quad \lambda=\sqrt{\frac{1-\sqrt{1-4 \epsilon^{2}}}{2 \epsilon^{2}}}=1+O\left(\epsilon^{2}\right) \text {. }
$$

The solution $u$ of (34) is given by:

$$
u=A e^{\Lambda x}+B e^{-\Lambda x}+C e^{\lambda x}+D e^{-\lambda x}
$$

where $A, B, C, D$ satisfy:

$$
\left\{\begin{array}{cl}
A+B+C+D & =R \\
\Lambda(A-B)+\lambda(C-D) & =0 \\
\Lambda^{2}(A+B)+\lambda^{2}(C+D) & =\frac{P}{\epsilon} \\
\Lambda^{3}(A-B)+\lambda^{3}(C-D) & =-\frac{Q}{\epsilon^{2}}
\end{array}\right.
$$

The exact solutions and asymptotic approximations for these constants are:

$$
\left\{\begin{array}{l}
A=\frac{\epsilon P-\Lambda^{-1} Q-\epsilon^{2} \lambda^{2} R}{2 \sqrt{1-4 \epsilon^{2}}}=\frac{\epsilon(P-Q-\epsilon R)}{2}+O\left(\epsilon^{3}\right)=o(\epsilon) \\
B=\frac{\epsilon P+\Lambda^{-1} Q-\epsilon^{2} \lambda^{2} R}{2 \sqrt{1-4 \epsilon^{2}}}=\frac{\epsilon(P+Q-\epsilon R)}{2}+O\left(\epsilon^{3}\right)=\epsilon+o(\epsilon) \\
C=\frac{\epsilon^{2}\left(\Lambda^{2} R-\frac{P}{\epsilon}+\frac{Q}{\lambda \epsilon^{2}}\right)}{2 \sqrt{1-4 \epsilon^{2}}}=\frac{-\epsilon P+Q+R}{2}+O\left(\epsilon^{2}\right)=\frac{1}{2}+o(1) \\
D=\frac{\epsilon^{2}\left(\Lambda^{2} R-\frac{P}{\epsilon}-\frac{Q}{\lambda \epsilon^{2}}\right)}{2 \sqrt{1-4 \epsilon^{2}}}=\frac{-\epsilon P-Q+R}{2}+O\left(\epsilon^{2}\right)=-\frac{1}{2}+o(1) .
\end{array}\right.
$$

Now, if $u_{x}(l)=0$, we have:

$$
\Lambda\left(A e^{\Lambda l}-B e^{-\Lambda l}\right)+\lambda\left(C e^{\lambda l}-D e^{-\lambda l}\right)=0 \quad \text { or } \quad A e^{\Lambda l}=B e^{-\Lambda l}-\lambda \Lambda^{-1}\left(C e^{\lambda l}-D e^{-\lambda l}\right) .
$$

Then the solution form (35) for $u$ becomes:

$$
u(x)=\left(B e^{-\Lambda l}-\lambda \Lambda^{-1}\left(C e^{\lambda l}-D e^{-\lambda l}\right)\right) e^{-\Lambda l} e^{\Lambda x}+B e^{-\Lambda x}+C e^{\lambda x}+D e^{-\lambda x}
$$

which leads to the following expressions:

$$
\left\{\begin{array}{l}
R^{\prime}=u(l)=2 B e^{-\Lambda l}+\left(C e^{\lambda l}+D e^{-\lambda l}\right)-\epsilon\left(C e^{\lambda l}-D e^{-\lambda l}\right)+O\left(\epsilon^{2}\right) \\
P^{\prime}=-\epsilon u_{x x}(l)=-\frac{2 B e^{-\Lambda l}}{\epsilon}+\left(C e^{\lambda l}-D e^{-\lambda l}\right)-\epsilon\left(C e^{\lambda l}+D e^{-\lambda l}\right)+O\left(\epsilon^{2}\right) \\
Q^{\prime}=-\epsilon^{2} u_{x x x}(l)=\left(C e^{\lambda l}-D e^{-\lambda l}\right)-\epsilon^{2}\left(C e^{\lambda l}-D e^{-\lambda l}\right)+O\left(\epsilon^{2}\right) .
\end{array}\right.
$$


Similar results can also be derived for the case $u_{x}<0$. Then we would write

$$
P=-\epsilon u_{x x}(0), \quad Q=\epsilon^{2} u_{x x x}(0) \quad \text { and } \quad P^{\prime}=\epsilon u_{x x}(l), \quad Q^{\prime}=\epsilon^{2} u_{x x x}(0)
$$

so that $P, P^{\prime}, Q, Q^{\prime}$ are all positive numbers.

The following classification of segments is crucial to our approach.

Definition 3.1. A segment $\left(c_{i}, c_{i+1}\right)$ is called long if $\left(c_{i+1}-c_{i}\right) \geq 10 \epsilon|\ln \epsilon|$. It is called short otherwise.

By means of (37), we now expand the formulas (39) so as to relate $\left(R^{\prime}, P^{\prime}, Q^{\prime}\right)$ to $(R, P, Q)$. The results are grouped into four categories.

Proposition 3.2. Let $Q=1+\alpha$ and $\nu^{2}=e^{-\frac{l}{\epsilon}}$. For any $c_{1}>0$, there exist $\epsilon_{*}$ and $l_{*}>0$ such that the following expressions hold for all $\epsilon<\epsilon_{*}$ :

Long positive segment. If $u_{x}>0$ and $10 \epsilon|\ln \epsilon| \leq l=L \leq l_{*}$, then

$$
\begin{aligned}
P & =Q+\epsilon R+O\left(\epsilon^{2}\right) \\
P^{\prime} & =Q^{\prime}-\epsilon R^{\prime}+O\left(\epsilon^{2}\right) \\
R^{\prime} & =R+L-2 \epsilon+\alpha L-2 \epsilon \alpha+\frac{L^{3}}{6}+\frac{R L^{2}}{2}-\epsilon R L+\frac{\alpha L^{3}}{6}+o\left|L^{3}\right|+O\left(\epsilon^{2}\right) \\
P^{\prime} & =Q+\left(R+\frac{L}{2}-\epsilon\right) L+\frac{\alpha L^{2}}{2}-\epsilon \alpha L-\epsilon R^{\prime}+o\left|L^{3}\right|+O\left(\epsilon^{2}\right) \\
Q^{\prime} & =Q+\left(R+\frac{L}{2}-\epsilon\right) L+\frac{\alpha L^{2}}{2}-\epsilon \alpha L+o\left|L^{3}\right|+O\left(\epsilon^{2}\right) .
\end{aligned}
$$

Long negative segment. If $u_{x}<0$ and $10 \epsilon|\ln \epsilon| \leq l=L \leq l_{*}$, then

$$
\begin{aligned}
P & =Q-\epsilon R+O\left(\epsilon^{2}\right) \\
P^{\prime} & =Q^{\prime}+\epsilon R^{\prime}+O\left(\epsilon^{2}\right) \\
R^{\prime} & =R-L+2 \epsilon-\alpha L+2 \epsilon \alpha-\frac{L^{3}}{6}+\frac{R L^{2}}{2}-\epsilon R L-\frac{\alpha L^{3}}{6}+o\left|L^{3}\right|+O\left(\epsilon^{2}\right) \\
P^{\prime} & =Q-\left(R-\frac{L}{2}+\epsilon\right) L+\frac{\alpha L^{2}}{2}-\epsilon \alpha L+\epsilon R^{\prime}+o\left|L^{3}\right|+O\left(\epsilon^{2}\right) \\
Q^{\prime} & =Q-\left(R-\frac{L}{2}+\epsilon\right) L+\frac{\alpha L^{2}}{2}-\epsilon \alpha L+o\left|L^{3}\right|+O\left(\epsilon^{2}\right) .
\end{aligned}
$$

Short positive segment. If $u_{x}>0$ and $l \leq 10 \epsilon|\ln \epsilon|$, then

$$
\begin{aligned}
P & =Q-2 Q \nu^{2}+\epsilon R+O\left(\epsilon^{2}\right)+o\left(\nu^{2}\right) \\
P^{\prime} & =Q^{\prime}-2 Q \nu^{2}-\epsilon R^{\prime}+O\left(\epsilon^{2}\right)+o\left(\nu^{2}\right) \\
R^{\prime} & =R+l-2 \epsilon+\alpha l-2 \epsilon \alpha+\frac{R l^{2}}{2}-\epsilon R l+2 \epsilon \nu^{2}+o\left(\epsilon \nu^{2}\right)+O\left(\epsilon^{2}\right)+o\left(\nu^{2}\right) \\
P^{\prime} & =Q-2 Q \nu^{2}+\left(R+\frac{l}{2}-\epsilon\right) l+\frac{\alpha l^{2}}{2}-\epsilon \alpha l-\epsilon R^{\prime}+O\left(\epsilon^{2}\right)+o\left(\nu^{2}\right) \\
Q^{\prime} & =Q+\left(R+\frac{l}{2}-\epsilon\right) l+\frac{\alpha l^{2}}{2}-\epsilon \alpha l+O\left(\epsilon^{2}\right)+o\left(\nu^{2}\right) .
\end{aligned}
$$


Short negative segment. If $u_{x}<0$ and $l \leq 10 \epsilon|\ln \epsilon|$, then

$$
\begin{aligned}
P & =Q-2 Q \nu^{2}-\epsilon R+O\left(\epsilon^{2}\right)+o\left(\nu^{2}\right) \\
P^{\prime} & =Q^{\prime}-2 Q \nu^{2}+\epsilon R^{\prime}+O\left(\epsilon^{2}\right)+o\left(\nu^{2}\right) \\
R^{\prime} & =R-l+2 \epsilon-\alpha l+2 \epsilon \alpha+\frac{R l^{2}}{2}-\epsilon R l-2 \epsilon \nu^{2}+o\left(\epsilon \nu^{2}\right)+O\left(\epsilon^{2}\right)+o\left(\nu^{2}\right) \\
P^{\prime} & =Q-2 Q \nu^{2}-\left(R-\frac{l}{2}+\epsilon\right) l+\frac{\alpha l^{2}}{2}-\epsilon \alpha l+\epsilon R^{\prime}+O\left(\epsilon^{2}\right)+o\left(\nu^{2}\right) \\
Q^{\prime} & =Q-\left(R-\frac{l}{2}+\epsilon\right) l+\frac{\alpha l^{2}}{2}-\epsilon \alpha l+O\left(\epsilon^{2}\right)+o\left(\nu^{2}\right) .
\end{aligned}
$$

We make the following remarks about the above expansions:

1. if $l \geq 10 \epsilon|\ln \epsilon|$, then $e^{-\frac{l}{\epsilon}} \ll O\left(\epsilon^{2}\right)$;

2. for all $\delta$, if $\mathcal{E}(u)$ is small enough, then $\nu^{2}=e^{-\frac{l}{\epsilon}}<\delta$ (see (33));

3. $e^{-\Lambda l}=\nu^{2}+o\left(\nu^{2}\right)$;

4. the expressions (41), (46), (51), and (56) come directly from (39) and are consistent with the formulas for $R^{\prime}, P^{\prime}$, and $Q^{\prime}$ (such as (42), (43), (44) and so forth);

5. not all the terms are relevant in the actual analysis. In fact, we just need to keep the terms of order up to $L^{2}, l^{2}$, and $\nu^{2}$.

As a first application of the above solution formula, we have the following existence and uniqueness result for the solution of the initial-final value problem (34). Hence the functions $P(x, l)$ 's are well defined in (2). The proof is elementary [18].

Proposition 3.3. There exist $l_{*}, \alpha, \beta>0$ such that for all $0<l \leq l_{*}$ and $Q, Q^{\prime}$ satisfying $\left|Q-Q^{\prime}\right| \leq \alpha l$ and $|Q+1| \leq \beta$ the following boundary value problem

$$
\begin{aligned}
\epsilon^{2} u_{x x x x}-u_{x x}+u=0 & \text { for } x \in(0, l) \\
u_{x}(0)=0 ; & u_{x}(l)=0 ; \\
\epsilon^{2} u_{x x x}(l)=Q, & \epsilon^{2} u_{x x x}(0)=Q^{\prime}
\end{aligned}
$$

has a unique solution $u$. In addition, $u_{x}(x)>0$ for $x \in(0, l)$.

Before leaving this section, we introduce some notations to be used in all of the following analysis and figures. The function $u$ always refers to a function from $\mathcal{Z}$ which solves (9) in the sense of Definition 2.2. The triples $\left(R_{i}, \pm P_{i}, \pm Q_{i}\right)$ and $\left(R_{i}^{\prime}, \pm P_{i}^{\prime}, \pm Q_{i}^{\prime}\right)$ denote the values of $\left(u, \epsilon u_{x x}, \epsilon^{2} u_{x x x}\right)$ at $c_{i}^{+}$and $c_{i+1}^{-}$. The \pm are chosen according to the sign of $u_{x}$ in $\left(c_{i}, c_{i+1}\right)$. With this notation, we then have

$$
R_{i}^{\prime}=R_{i+1}, \quad P_{i}^{\prime}=P_{i+1} \text { and } Q_{i}^{\prime}+Q_{i+1}=2 .
$$

\section{Proof of Theorem 1.1}

In this section, we will prove the stability of periodic structures with long periods. The approach is similar to [12], page 199, and hence we will just outline the steps and state the key estimates.

By approximation, we can assume that $\varphi$ is $C^{\infty}$. In this case, we have the expression (17) for the second variation of $\mathcal{E}$ with respect to $\varphi$. In addition, the zeros $\left\{c_{i}(t)\right\}_{i=1}^{N-1}$ of $u_{x}(x)+t \varphi_{x}(x)$ are $C^{1}$ functions satisfying (16).

We first present the following result which essentially establishes the convexity property for the minimum energy value in a unit cell. 
Proposition 4.1. Let $P(x, l)$ be defined as in (2) and

$$
E(l)=\int_{-\frac{l}{2}}^{\frac{l}{2}} \epsilon^{2} P_{x x}^{2}+\left(P_{x}-1\right)^{2}+P^{2} \mathrm{~d} x .
$$

Then

1. $\int_{-\frac{l}{2}}^{\frac{l}{2}} \epsilon^{2} v_{x x}^{2}+\left(v_{x}-1\right)^{2}+v^{2} \mathrm{~d} x \geq E(l)$ where $v_{x}>0$ for $x \in\left(-\frac{l}{2}, \frac{l}{2}\right)$ and $v_{x}\left( \pm \frac{l}{2}\right)=0$.

2. There exist constants $C_{L}<10, l_{*}$ and $\epsilon_{*}>0$ such that for any $0<\epsilon<\epsilon_{*}$, if $C_{L} \epsilon|\ln \epsilon|<l<l_{*}$, then for some constant $C$,

$$
\begin{array}{r}
\left|E(l)-\left(2 \epsilon+\frac{l^{3}}{12}\right)\right| \leq C l^{3}\left(l^{2}+\frac{\epsilon}{l}\right) \\
\left|E^{\prime}(l)-\frac{l^{2}}{4}\right| \leq C l^{2}\left(l^{2}+\frac{\epsilon}{l}\right) \\
\left|E^{\prime \prime}(l)-\frac{l}{2}\right| \leq C l\left(l^{2}+\frac{\epsilon}{l}\right) .
\end{array}
$$

This proposition is basically the same as [12], Theorems 4.2(i), 5.1. In the current work, the proof can be carried out in a more elementary way due to the presence of explicit solution formulas [18].

Now Theorem 1.1 follows easily as shown below. For small enough $t$,

$$
\mathcal{E}(u+t \varphi) \geq \sum_{i=0}^{N-1} E\left(l_{i}(t)\right) \text { where } l_{i}(t)=c_{i+1}(t)-c_{i}(t)
$$

As $\mathcal{E}(u)=\sum_{i} E\left(l_{i}(0)\right)$, the above leads to

$$
\left.\frac{\mathrm{d}}{\mathrm{d} t} \mathcal{E}(u+t \varphi)\right|_{t=0}=\left.\sum_{i} \frac{\mathrm{d}}{\mathrm{d} t} E\left(l_{i}(t)\right)\right|_{t=0} \text { and }\left.\frac{\mathrm{d}^{2}}{\mathrm{~d} t^{2}} \mathcal{E}(u+t \varphi)\right|_{t=0} \geq\left.\sum_{i} \frac{\mathrm{d}^{2}}{\mathrm{~d} t^{2}} E\left(l_{i}(t)\right)\right|_{t=0} .
$$

Since

we have

$$
\frac{\mathrm{d}}{\mathrm{d} t} E\left(l_{i}(t)\right)=E^{\prime}\left(l_{i}(t)\right) \dot{l}_{i}(t) \text { and } \frac{\mathrm{d}^{2}}{\mathrm{~d} t^{2}} E\left(l_{i}(t)\right)=E^{\prime \prime}\left(l_{i}(t)\right)\left(\dot{l}_{i}(t)\right)^{2}+E^{\prime}\left(l_{i}(t)\right) \ddot{l}_{i}(t)
$$

$$
\begin{aligned}
\left.\frac{\mathrm{d}^{2}}{\mathrm{~d} t^{2}} \mathcal{E}(u+t \varphi)\right|_{t=0} & \geq \sum_{i} E^{\prime \prime}\left(l_{i}\right)\left(i_{i}(0)\right)^{2}+E^{\prime}\left(l_{i}\right) \ddot{l}_{i}(0) \\
& =E^{\prime \prime}(l) \sum_{i}\left(i_{i}(0)\right)^{2}+E^{\prime}(l) \sum_{i}\left(\ddot{l}_{i}(0)\right) \quad\left(\text { since } l_{i}(0)=l\right) \\
& =E^{\prime \prime}(l) \sum_{i}\left(i_{i}(0)\right)^{2} \quad\left(\text { since } \sum_{i} l_{i}=1\right) \\
& \geq M l \sum_{i}\left(i_{i}(0)\right)^{2} \quad(\text { by }(64))
\end{aligned}
$$

where the constant $M$ can be chosen to be close to $\frac{1}{2}$ (independent of $\epsilon$ and $l$ ). The desired result follows by using the facts that $\dot{l}_{i}(0)=\dot{c}_{i+1}(0)-\dot{c}_{i}(0)$ and also $(16)$. 


\section{Proof of Theorem 1.2}

The essence of the proof is to analyze the patterns consisting of both long and short segments and show that they have negative second variations. This is achieved by careful consideration of the interactions and matchings between long and short segments. We rely heavily on the formulas derived in Section 3 .

To prepare for the proof, we first show that there must be at least one segment of length longer than $5 \bar{L}$ where $\bar{L}=10 \epsilon|\ln \epsilon|$ is the length of a long segment (see Def. 3.1). For if otherwise, by Lemma 2.8, we would have

$$
\begin{aligned}
\mathcal{E}(u) & \geq(\text { no. of segments }) \times(\text { lower bound for the energy in each segment }) \\
& >\frac{1}{5 \bar{L}} \frac{3}{4} \epsilon=\frac{3}{200|\ln \epsilon|}
\end{aligned}
$$

contradicting the smallness assumption on the energy (4).

Now let $\left(c_{i}, c_{i+1}\right)$ be any one of the segments with length $\geq 5 \bar{L}$. Assume for the moment this segment is not at the boundary. Consider the two adjacent segments $\left(c_{i-1}, c_{i}\right)$ and $\left(c_{i+1}, c_{i+2}\right)$. The following three cases can happen:

1. If both of them are short, we call the collection of segments $\left\{\left(c_{j}, c_{j+1}\right): j=i-1, i, i+1\right\}$ SLS.

2. If only one of them is short, we call the same collection of segments SLL or LLS.

3. If both of them are long, without loss of generality, we consider the segments to the right of $\left(c_{i+1}, c_{i+2}\right)$.

We keep searching to the right until we find a short segment $\left(c_{k}, c_{k+1}\right.$ ). By Proposition 5.2 (proved later), the segments $\left\{\left(c_{j}, c_{j+1}\right): j=i, \ldots k-2\right\}$ have roughly the same length. Now if $c_{k}-c_{k-1} \geq$ $\frac{1}{2}\left(c_{k-1}-c_{k-2}\right) \geq 2 \bar{L}$, then we consider the collection of segments $\left\{\left(c_{j}, c_{j+1}\right): j=k-2, k-1, k\right\}$ and call it LLS. If otherwise, we then consider the collection $\left\{\left(c_{j}, c_{j+1}\right): j=k-3, k-2, k-1\right\}$ and still regard it as LLS. (Note that in this case, the segment $\left(c_{k-1}, c_{k}\right)$ is not yet known to be short according to Def. 3.1.)

If the initial long segment $\left(c_{i}, c_{i+1}\right)$ is at the boundary, we can reflect the function $u$ across the boundary point (which is admissible due to the boundary conditions). Now the segment will have at least one long segments as a neighbor. Hence only the cases 2 or 3 above will arise.

In view of the above discussion, if $u$ has both long and short segments, then it must contain a pattern of SLL, LLS or SLS. Hence Theorem 1.2 will be proved once we establish the following claims:

Claim I. If $u$ has a chain of adjacent long segments, then a sub-chain of them must have roughly the same length.

Claim II. If $u$ contains any of the patterns LLS, SLL or SLS, then it is unstable.

Claim III. If $u$ is a (nearly periodic) pattern with low enough energy with only long segments, then it is actually periodic.

We now proceed to prove the claims. Their precise statements will be given along the proof.

\subsection{Proof of Claim I}

Lemma 5.1. If both $\left(c_{i-1}, c_{i}\right)$ and $\left(c_{i}, c_{i+1}\right)$ are long segments, then

$$
Q_{i-1}^{\prime}=1+O\left(\epsilon^{2}\right) \quad \text { and } \quad Q_{i}=1+O\left(\epsilon^{2}\right) .
$$

Proof. Consider the point $c_{i}$. By (41) and (45), we have

$$
P_{i-1}^{\prime}=Q_{i-1}^{\prime}-\epsilon R_{i-1}^{\prime}+O\left(\epsilon^{2}\right) \text { and } P_{i}=Q_{i}-\epsilon R_{i}+O\left(\epsilon^{2}\right) .
$$

Since $P_{i-1}^{\prime}=P_{i}$ and $R_{i-1}^{\prime}=R_{i}$, it follows that $Q_{i-1}^{\prime}-Q_{i}=O\left(\epsilon^{2}\right)$. This, together with the continuity condition (13) $-Q_{i-1}^{\prime}+Q_{i}=2$ - gives the desired statement. 
Proposition 5.2. If $\left\{\left(c_{i}, c_{i+1}\right), i=m, m+1, \ldots, n\right\}$ are all long segments, then there exists an $L_{\epsilon}>0$ such that

$$
\begin{aligned}
& \quad|| R_{i}\left|-\left(\frac{L_{\epsilon}}{2}-\epsilon\right)\right|=o(\epsilon) \quad \text { for } i=m+1, \ldots n \\
& \text { and } L_{i}=c_{i+1}-c_{i}=L_{\epsilon}+o\left(L_{\epsilon}^{2}\right)+o(\epsilon) \text { for } i=m+1, \ldots n-1 .
\end{aligned}
$$

Proof. First, by the previous lemma, we have $Q_{i}=1+O\left(\epsilon^{2}\right)$ for $i=m+1, \ldots, n$. Then (44) and (49) lead to

$$
R_{i}= \pm \frac{L_{i}}{2} \mp \epsilon+o\left(L_{i}^{2}\right)+o(\epsilon), \quad i=m+1, \ldots, n
$$

Hence we must have $\left|R_{i}\right| \gg O(\epsilon)$ for the same range of the $i$ 's.

Next, by setting $x=c_{i+1}$ into (23), we obtain

$$
\epsilon^{2} u_{x x}^{2}\left(c_{i+1}\right)-\epsilon^{2} u_{x x}^{2}\left(c_{i}\right)=u^{2}\left(c_{i+1}\right)-u^{2}\left(c_{i}\right) \quad \text { or } \quad P_{i+1}^{2}-P_{i}^{2}=R_{i+1}^{2}-R_{i}^{2}
$$

Upon summing over the $i$ 's, it holds that

$$
P_{j}^{2}-P_{i}^{2}=R_{j}^{2}-R_{i}^{2}
$$

By restricting $m+1 \leq i, j \leq n$, we can again invoke Lemma 5.1 which together with (40), (41), (45) and (46) gives

$$
\left(1 \pm \epsilon R_{j}\right)^{2}-\left(1 \pm \epsilon R_{i}\right)^{2}=R_{j}^{2}-R_{i}^{2}+O\left(\epsilon^{2}\right)
$$

If $R_{i} R_{j}>0$, then

$$
\begin{aligned}
\left(1+\epsilon R_{j}\right)^{2}-\left(1+\epsilon R_{i}\right)^{2} & =R_{j}^{2}-R_{i}^{2}+O\left(\epsilon^{2}\right) \\
2 \epsilon\left(R_{j}-R_{i}\right) & =\left(R_{j}-R_{i}\right)\left(R_{j}+R_{i}\right)+O\left(\epsilon^{2}\right) \\
R_{j}-R_{i} & =\frac{O\left(\epsilon^{2}\right)}{R_{i}+R_{j}+2 \epsilon}=o(\epsilon) \quad\left(\text { since }\left|R_{i}\right|,\left|R_{j}\right| \gg \epsilon\right) .
\end{aligned}
$$

Similarly, if $R_{i} R_{j}<0$, then

$$
R_{j}+R_{i}=\frac{O\left(\epsilon^{2}\right)}{R_{i}-R_{j}+2 \epsilon}=o(\epsilon)
$$

To conclude (66), we can just take $L_{\epsilon}$ to be any of the $2\left(\left|R_{i}\right|+\epsilon\right)$ 's. Statement (67) would also follow from (68) which now says $L_{i}=L_{\epsilon}+o\left(L_{i}^{2}\right)+o(\epsilon)$.

The following corollary is interesting in its own right even though it is not used in the later parts of our proof. It demonstrates that just the consideration of the first variation can already lead to some strong conclusion.

Corollary 5.3 (nearly periodic structures). If $u \in \mathcal{Z}$ has only long segments, then it is nearly periodic in the sense that there exists $R_{\epsilon}$ and $L_{\epsilon}$ such that (66) and (67) hold for all $i=0,1, \ldots N-1$.

Proof. We can extend the conclusion to the boundary segments because it automatically holds that $\epsilon^{2} u_{x x x}=$ $\pm 1+O\left(\epsilon^{2}\right)$ at the boundary points. This is what is actually needed in the proof. 


\subsection{Proof of Claim II}

Without loss of generality, it suffices to consider the following categories of patterns for $u_{x}$ (Figs. 4 and 5 ):

\section{LLS (Long-Long-Short).}

$|E A|=L_{1} \geq 10 \epsilon|\ln \epsilon| ;|A B|=L \geq 10 \epsilon|\ln \epsilon|$
$|B C|=l \leq \frac{1}{2}|A B|$

\section{SLS (Short-Long-Short).}

$$
\begin{gathered}
|E A|=m ; \quad|A B|=L \geq 10 \epsilon|\ln \epsilon| \\
|B C|=l ;|E A|,|B C| \leq \frac{|A B|}{2}
\end{gathered}
$$

$$
\begin{array}{cc}
\left(R_{0}, P_{0}^{u_{x}}, Q_{0}\right) & \left(R_{2}, P_{2}, Q_{2}\right) \\
\hline E{ }_{\left(R_{1}^{m}, P_{1}, Q_{1}\right)}^{A} Q^{L} & \left(R_{3}, P_{3}, Q_{3}\right)
\end{array}
$$

Figure 5. An SLS pattern.

FiguRE 4. An LLS pattern.

We will produce test function $\varphi^{\prime}$ s such that $\partial^{2} \mathcal{E}(u, \varphi)<0$ for the above patterns. They are constructed by moving the interfaces and are given precisely in the next section.

\subsubsection{Construction of test functions}

Consider the following second order (Allen-Cahn) equation:

$$
\begin{gathered}
\epsilon^{2} U_{x x x}=\frac{W^{\prime}\left(U_{x}\right)}{2} \text { or equivalently, } \epsilon^{2} U_{x x x}=U_{x}-1 \\
\text { such that } U_{x}( \pm l)=0 \text { and } U_{x}>0 \text { for }|x| \leq l .
\end{gathered}
$$

The solution, considered as a function of $x$ and $l$, is given by:

$$
U_{x}(x, l)=1-\frac{e^{\frac{x}{\epsilon}}+e^{-\frac{x}{\epsilon}}}{e^{\frac{l}{\epsilon}}+e^{-\frac{l}{\epsilon}}}
$$

Let $\nu^{2}=e^{-\frac{2 l}{\epsilon}}-$ note that $2 l$ is the length of the segment. Associated with the above function are the following quantities:

$$
\begin{gathered}
U_{x}(-l, l)=0, \quad U_{x}(l, l)=0 \\
U_{x x}(-l, l)=\frac{1}{\epsilon}\left(\frac{1-\nu^{2}}{1+\nu^{2}}\right), \quad U_{x x}(l, l)=-\frac{1}{\epsilon}\left(\frac{1-\nu^{2}}{1+\nu^{2}}\right) \\
U_{x l}(-l, l)=\frac{1}{\epsilon}\left(\frac{1-\nu^{2}}{1+\nu^{2}}\right), \quad U_{x l}(l, l)=\frac{1}{\epsilon}\left(\frac{1-\nu^{2}}{1+\nu^{2}}\right) \\
U_{x x x}(-l, l)=-\frac{1}{\epsilon^{2}}, \quad U_{x x x}(l, l)=-\frac{1}{\epsilon^{2}} \\
U_{x x l}(-l, l)=-\frac{1}{\epsilon^{2}}\left(\frac{1-\nu^{2}}{1+\nu^{2}}\right)^{2}, \quad U_{x x l}(l, l)=\frac{1}{\epsilon^{2}}\left(\frac{1-\nu^{2}}{1+\nu^{2}}\right)^{2}
\end{gathered}
$$

which will be used to compute the second variations. 
Making use of the above $U_{x}(x, l)$, we introduce two types of test functions which mimic the movements of the interfaces (Figs. 6 and 7):

\section{Type one $-F_{x}(x, l)-$ movement of one interface}
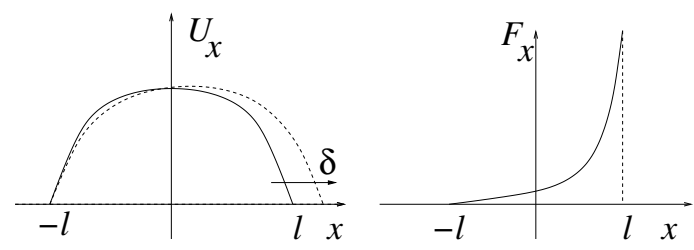

Figure 6. Type one variation.

$$
F_{x}(x, l)=\lim _{\delta \rightarrow 0} \frac{U_{x}\left(x-\frac{\delta}{2}, l+\frac{\delta}{2}\right)-U_{x}(x, l)}{\delta}=-\frac{U_{x x}(x, l)}{2}+\frac{U_{x l}(x, l)}{2} .
$$

Then $F$ satisfies the following estimates:

$$
\begin{gathered}
F_{x}(-l, l)=0, \quad F_{x}(l, l)=\frac{1}{\epsilon}\left(\frac{1-\nu^{2}}{1+\nu^{2}}\right) \\
\int_{-l}^{l} \epsilon^{2} F_{x x}^{2}+F_{x}^{2} \mathrm{~d} x=\frac{1}{\epsilon}\left(1-4 \nu^{2}+o\left(\nu^{2}\right)\right) \\
F(l, l)-F(-l, l)=1-4 \nu^{2}+o\left(\nu^{2}\right) .
\end{gathered}
$$

\section{Type two $-G_{x}(x, l)-$ translation of two adjacent interfaces}

Similarly, $G$ satisfies:

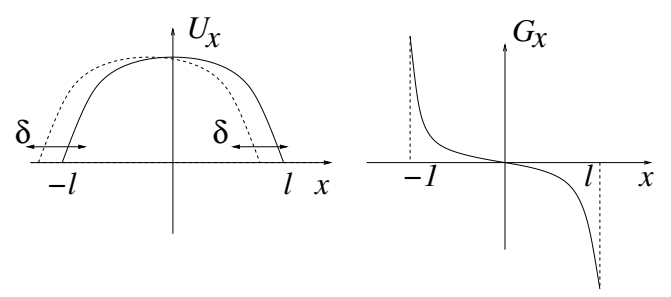

Figure 7. Type two variation.

$$
G_{x}(x, l)=\lim _{\delta \rightarrow 0} \frac{U_{x}(x \pm \delta, l)-U_{x}(x, l)}{\delta}= \pm U_{x x}(x, l) .
$$

$$
\begin{gathered}
G_{x}(-l, l)= \pm \frac{1}{\epsilon}\left(\frac{1-\nu^{2}}{1+\nu^{2}}\right), \quad G_{x}(l, l)=\mp \frac{1}{\epsilon}\left(\frac{1-\nu^{2}}{1+\nu^{2}}\right) \\
\int_{-l}^{l} \epsilon^{2} G_{x x}^{2}+G_{x}^{2} \mathrm{~d} x=\frac{2}{\epsilon}\left(1-2 \nu^{2}+o\left(\nu^{2}\right)\right) \\
G(l, l)-G(-l, l)=0 .
\end{gathered}
$$

A bit of motivation for the following analysis is in place. From (69) and (71), the negativity of the second variations comes from $-\nu^{2}$. So our goal is to characterize $\nu^{2}$ as accurately as possible. It turns out that if a short segment is adjacent to a long segment, then its length cannot be arbitrary. This is due to some intricate interactions between the long and short segments. We will use the results of Proposition 3.2 to provide some lower bounds for the values of $\nu^{2}$. It is unfortunate that the present approach requires the consideration of a large number of cases.

We now proceed to analyze the patterns LLS and SLS. 


\subsubsection{Instability of $L L S$}

We refer to Figure 4. Consider the point $A$. By (40) and (65), we have

$$
P_{0}=1+\epsilon R_{0}+O\left(\epsilon^{2}\right) \quad \text { and } \quad Q_{0}=1+O\left(\epsilon^{2}\right) .
$$

Using (43) and (44) for the propagation map $\left(R_{0}, P_{0}, Q_{0}\right) \longrightarrow_{L}\left(R_{0}^{\prime}, P_{0}^{\prime}, Q_{0}^{\prime}\right)$, at $B$, we have

$$
\begin{aligned}
& P_{0}^{\prime}=1+\left(R_{0}+\frac{L}{2}-\epsilon\right) L-\epsilon R_{1}+O\left(L^{3}\right)+O\left(\epsilon^{2}\right) \\
& Q_{0}^{\prime}=1+\left(R_{0}+\frac{L}{2}-\epsilon\right) L+O\left(L^{3}\right)+O\left(\epsilon^{2}\right) .
\end{aligned}
$$

Next consider the triples $\left(R_{0}^{\prime}, P_{0}^{\prime}, Q_{0}^{\prime}\right)$ and $\left(R_{1}, P_{1}, Q_{1}\right)$ associated with the point $B$. Let $\nu^{2}=e^{-\frac{l}{\epsilon}}$. By $(41)$ and (55), the following hold:

$$
\begin{aligned}
& P_{0}^{\prime}=Q_{0}^{\prime}-\epsilon R_{1}+O\left(\epsilon^{2}\right) \\
& P_{1}=Q_{1}-2 Q_{1} \nu^{2}-\epsilon R_{1}+O\left(\epsilon \nu^{2}\right)+O\left(\epsilon^{2}\right)
\end{aligned}
$$

which together with $P_{0}^{\prime}=P_{1}$ and $Q_{0}^{\prime}+Q_{1}=2$ lead to

$$
\begin{aligned}
& Q_{0}^{\prime}=1-\nu^{2}+o\left(\nu^{2}\right)+O\left(\epsilon^{2}\right) \\
& Q_{1}=1+\nu^{2}+o\left(\nu^{2}\right)+O\left(\epsilon^{2}\right) .
\end{aligned}
$$

By substituting (79) into (77) and comparing (78) and (75), we have

$$
\begin{aligned}
& P_{1}=1-\nu^{2}-\epsilon R_{1}+o\left(\nu^{2}\right)+O\left(\epsilon^{2}\right) \\
& \nu^{2}=-\left(R_{0}+\frac{L}{2}-\epsilon\right) L+O\left(L^{3}\right)+o\left(\nu^{2}\right)+O\left(\epsilon^{2}\right) .
\end{aligned}
$$

We now consider the following three subcases..

Case of $\mathbf{L L S}_{\mathbf{1}}$. Assume $R_{0} \leq-\frac{2}{3} L$. By (81), this assumption leads to

$$
\nu^{2} \geq\left(\frac{2}{3}-\frac{1}{2}\right) L^{2}+o\left(L^{2}\right) \geq \frac{L^{2}}{7}
$$

Now vary the pattern by moving the interfaces at $A$ and $B$ as shown in Figure 8.

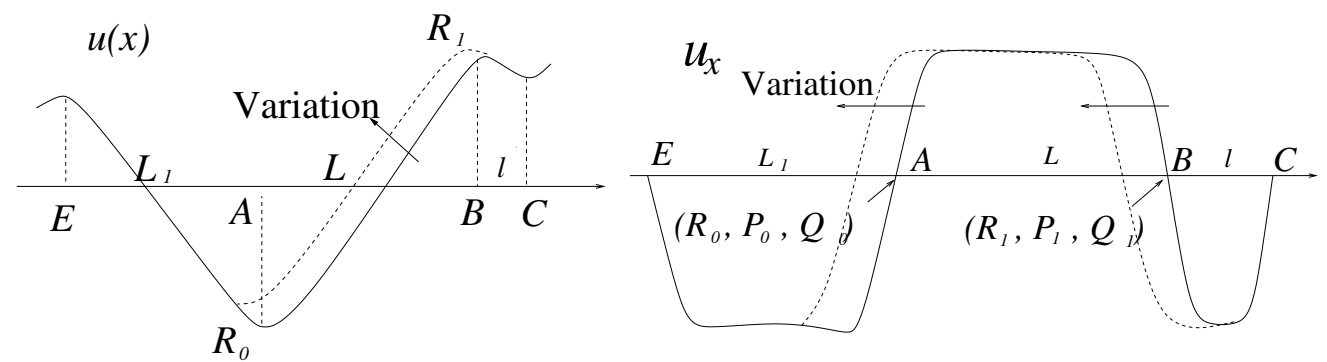

FIGURE 8. Variation of pattern $\mathbf{L L S}_{\mathbf{1}}$. 
This corresponds to the test function:

$$
\Psi_{x}= \begin{cases}F_{x}\left(x-\frac{E+A}{2}, \frac{A-E}{2}\right) & x \in(E, A) \\ G_{x}\left(x-\frac{A+B}{2}, \frac{B-A}{2}\right) & x \in(A, B) \\ -\left(\frac{1+\nu^{2}}{1-\nu^{2}}\right) F_{x}\left(-x+\frac{B+C}{2}, \frac{C-B}{2}\right) & x \in(B, C)\end{cases}
$$

(see the remark at the end of this case.) Now the quantity $\mathcal{D}(u, \Psi)$ equals:

$$
\begin{aligned}
\int_{E}^{C}\left(\epsilon^{2} \Psi_{x x}^{2}+\Psi_{x}^{2}\right) \mathrm{d} x-2 \frac{\Psi_{x}^{2}(A)}{\left|u_{x x}(A)\right|}-2 \frac{\Psi_{x}^{2}(B)}{\left|u_{x x}(B)\right|}= & \int_{E}^{A}\left(\epsilon^{2} F_{x x}^{2}+F_{x}^{2}\right) \mathrm{d} x+\int_{A}^{B}\left(\epsilon^{2} G_{x x}^{2}+G_{x}^{2}\right) \mathrm{d} x \\
& +\left(\frac{1+\nu^{2}}{1-\nu^{2}}\right)^{2} \int_{B}^{C}\left(\epsilon^{2} F_{x x}^{2}+F_{x}^{2}\right) \mathrm{d} x-2 \frac{1}{\epsilon} \frac{1}{P_{0}}-2 \frac{1}{\epsilon} \frac{1}{P_{1}} \\
= & \frac{1}{\epsilon}+\frac{2}{\epsilon}+\left(\frac{1+\nu^{2}}{1-\nu^{2}}\right)^{2} \frac{1}{\epsilon}\left(1-4 \nu^{2}\right) \\
& -\frac{2}{\epsilon} \frac{1}{1+\epsilon R_{0}+O\left(\epsilon^{2}\right)}-\frac{2}{\epsilon} \frac{1}{1-\nu^{2}-\epsilon R_{1}+o\left(\nu^{2}\right)+O\left(\epsilon^{2}\right)} \\
& =\frac{-2 \nu^{2}+2 \epsilon R_{0}-2 \epsilon R_{1}+o\left(\nu^{2}\right)+O\left(\epsilon^{2}\right)}{\epsilon}
\end{aligned}
$$

where in the above computation, we have used (73) and (80). By the lower bound (82) for $\nu^{2}$ and the fact that $\left|R_{1}-R_{0}\right|=L+o(L)$, we have

$$
\mathcal{D}(u, \Psi) \leq-\frac{L^{2}}{7 \epsilon}
$$

Now $\Psi(x) \approx 1$ for $x \in(A, B)$ and $\approx 0$ for $x \in(E, A) \cup(B, C)$. So $\int_{E}^{C} \Psi^{2} \mathrm{~d} x=L+o(L)$. Hence we have

$$
\partial^{2} \mathcal{E}(u, \Psi)=\mathcal{D}(u, \Psi)+\int_{E}^{C} \Psi^{2} \mathrm{~d} x=-\frac{L^{2}}{7 \epsilon}+L+o(L)<0 !
$$

proving that the pattern $\mathrm{LLS}_{1}$ is unstable.

\section{Remark about the test function $\Psi$ (83).}

1. The extra multiplicative factor for $F_{x}$ in the segment of $(B, C)$ is to make $\Psi_{x}$ continuous with a common value of $\frac{1}{\epsilon}$ at $A$ and $B$. In this way, the continuity of $\Psi$ holds up to error terms consisting of $e^{-\frac{L}{\epsilon}}$ which are transcendentally small compared with $\nu^{2}$ and hence can be ignored.

2. By $(70), \Psi(B)-\Psi(C)=1-O\left(\nu^{2}\right) \neq 1$, so an extra piece of perturbation needs to be added to $\Psi_{x}$ in order to make $\Psi(E)=0$ and $\Psi(C)=0$. This can be done by choosing an appropriate function $g_{x}(x)$ satisfying:

$$
\left\|g_{x}\right\|_{\infty} \leq \frac{O\left(\nu^{2}\right)}{L}, \quad\left\|g_{x x}\right\|_{\infty} \leq \frac{O\left(\nu^{2}\right)}{L^{2}} \quad \text { and } \operatorname{spt}\left(g_{x}\right) \subset(A, B)
$$

Note that $\left\|\Psi_{x}\right\|_{\infty}$ is transcendentally small near the center region of the long segment $(A, B)$, the error introduced by $g_{x}$ can be bounded by:

$$
\int_{A}^{B} \epsilon^{2} g_{x x}^{2}+g_{x}^{2} \mathrm{~d} x \leq \epsilon^{2} \times \frac{\nu^{4}}{L^{4}} \times L+\frac{\nu^{4}}{L^{2}} \times L=\frac{1}{\epsilon} o\left(\nu^{2}\right) .
$$

Compared with the expression $(84)$ for $\mathcal{D}(u, \Psi)$, the above is within the range of acceptable error for our analysis. Hence we can in effect ignore $g_{x}$. 
We will not repeat the above remarks for the remaining analysis.

Case of $\mathbf{L L S}_{\mathbf{2}}$. Assume $R_{0} \geq-\frac{2}{3} L$ and $Q_{2}=1+O\left(\epsilon^{2}\right)$. (The reason for introducing this and the following cases is that we might not have (82) - this can actually happen (see Sect. B). Thus we cannot directly deduce the same negativity for $\mathcal{D}(u, \Psi)$ as in (85). To tackle this, we further consider the pattern to the right of LLS.)

First, just the assumption on $R_{0}$ and the fact that $l \leq \frac{L}{2}$ imply:

$$
\left(R_{1}-\frac{l}{2}+\epsilon\right) l=\left(R_{0}+L+o(L)-\frac{l}{2}+\epsilon\right) l \geq\left(\frac{L}{3}-\frac{L}{4}+o(L)\right) l \geq \frac{L l}{13} .
$$

Consider the point $C$. In view of (59) and (79), it holds that:

$$
Q_{1}^{\prime}=1+\nu^{2}-\left(R_{1}-\frac{l}{2}+\epsilon\right) l+o\left(\nu^{2}\right)+O\left(\epsilon^{2}\right) .
$$

Since $Q_{1}^{\prime}=2-Q_{2}$ which equals $1+O\left(\epsilon^{2}\right)$ by the assumption of this case, the above leads to the following lower bound:

$$
\nu^{2}=\left(R_{1}-\frac{l}{2}+\epsilon\right) l+o\left(\nu^{2}\right)+O\left(\epsilon^{2}\right) \geq \frac{L l}{13} .
$$

Now using the same test function (83) and reasoning as in $\mathrm{LLS}_{1}$, we have:

$$
\mathcal{D}(u, \Psi)=\frac{-2 \nu^{2}+2 \epsilon R_{0}-2 \epsilon R_{1}+o\left(\nu^{2}\right)+O\left(\epsilon^{2}\right)}{\epsilon} \leq-\frac{L l}{7 \epsilon} \quad(\text { note: } l \gg \epsilon) .
$$

Thus it holds that

so that this case is also unstable.

$$
\partial^{2} \mathcal{E}(u, \Psi) \leq-\frac{L l}{7 \epsilon}+L+o(L)<0
$$

Case of LLSS. Assume $R_{0} \geq-\frac{2 L}{3}$ and there is a short segment $(C, D)$ to the right of $(B, C)$. The configurations of $u$ and $u_{x}$ are shown in Figure 9 .
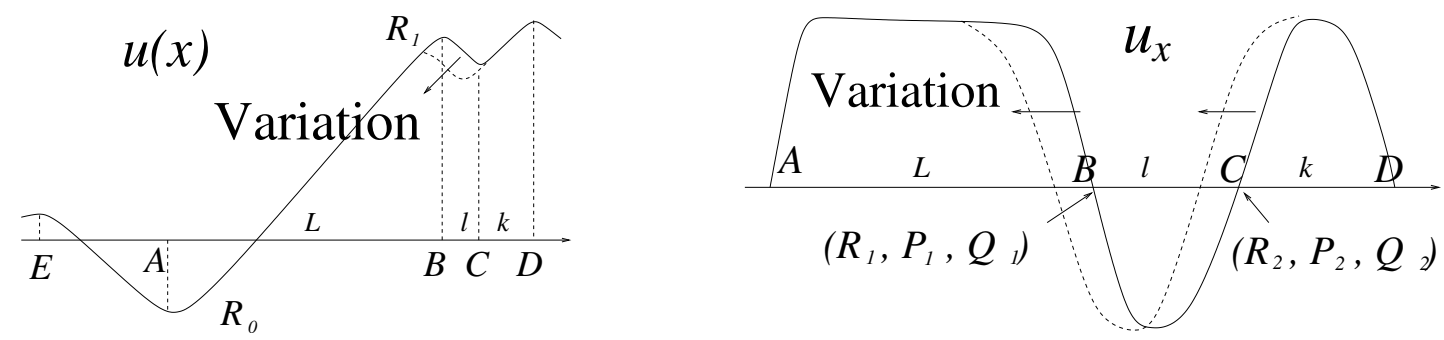

FIGURE 9. Variation of pattern LLSS.

Let $|C D|=k$ and $\mu^{2}=e^{-\frac{k}{\epsilon}}$. Consider the point $C$ again. Using the (58) and (79) associated with the propagation map from $B$ to $C$, we have

$$
\begin{aligned}
P_{1}^{\prime} & =Q_{1}-2 Q_{1} \nu^{2}-\left(R_{1}-\frac{l}{2}+\epsilon\right) l+\epsilon R_{2}+o\left(\nu^{2}\right)+O\left(\epsilon^{2}\right) \\
& =1-\nu^{2}-\left(R_{1}-\frac{l}{2}+\epsilon\right) l+\epsilon R_{2}+o\left(\nu^{2}\right)+O\left(\epsilon^{2}\right) .
\end{aligned}
$$

In addition, applying the map from $C$ to $D$ and by (50), we have:

$$
P_{2}=Q_{2}-2 Q_{2} \mu^{2}+\epsilon R_{2}+o\left(\mu^{2}\right) .
$$


Comparing (89) and (90) and using (87) and $Q_{1}^{\prime}+Q_{2}=2$ give:

$$
\begin{aligned}
Q_{2} & =1-\nu^{2}+\left(R_{1}-\frac{l}{2}+\epsilon\right) l+o\left(\nu^{2}\right)+O\left(\epsilon^{2}\right) \\
\mu^{2} & =\left(R_{1}-\frac{l}{2}+\epsilon\right) l+o\left(\nu^{2}\right)+o\left(\mu^{2}\right)+O\left(\epsilon^{2}\right)
\end{aligned}
$$

which lead to

$$
P_{2}=P_{1}^{\prime}=1-\nu^{2}-\mu^{2}+\epsilon R_{2}+o\left(\nu^{2}\right)+o\left(\mu^{2}\right)+O\left(\epsilon^{2}\right)
$$

Now vary the pattern by moving the interfaces at $B$ and $C$ as shown in Figure 9 . The test function $\Psi$ used is thus:

$$
\Psi_{x}= \begin{cases}-F_{x}\left(x-\frac{A+B}{2}, \frac{B-A}{2}\right) & x \in(A, B) \\ \left(\frac{1+\nu^{2}}{1-\nu^{2}}\right) G_{x}\left(x-\frac{B+C}{2}, \frac{C-B}{2}\right) & x \in(B, C) \\ \left(\frac{1+\mu^{2}}{1-\mu^{2}}\right) F_{x}\left(-x+\frac{C+D}{2}, \frac{D-C}{2}\right) & x \in(C, D) .\end{cases}
$$

In this case,

$$
\begin{aligned}
\mathcal{D}(u, \Psi) & =\int_{A}^{D}\left(\epsilon^{2} \Psi_{x x}^{2}+\Psi_{x}^{2}\right) \mathrm{d} x-2 \frac{\Psi_{x}^{2}(B)}{\left|u_{x x}(B)\right|}-2 \frac{\Psi_{x}^{2}(C)}{\left|u_{x x}(C)\right|} \\
& =\frac{1}{\epsilon}+\left(\frac{1+\nu^{2}}{1-\nu^{2}}\right)^{2} \frac{2}{\epsilon}\left(1-2 \nu^{2}\right)+\left(\frac{1+\mu^{2}}{1-\mu^{2}}\right)^{2} \frac{1}{\epsilon}\left(1-4 \mu^{2}\right)-\frac{2}{\epsilon} \frac{1}{P_{1}}-\frac{2}{\epsilon} \frac{1}{P_{2}} \\
& =\frac{1}{\epsilon}+\frac{2}{\epsilon}\left(1+2 \nu^{2}\right)+\frac{\left(1+o\left(\mu^{2}\right)\right)}{\epsilon}-\frac{2}{\epsilon} \frac{1}{1-\nu^{2}-\epsilon R_{1}}-\frac{2}{\epsilon} \frac{1}{1-\nu^{2}-\mu^{2}+\epsilon R_{2}} \\
& =\frac{-2 \mu^{2}-2 \epsilon R_{1}+2 \epsilon R_{2}+o\left(\mu^{2}\right)+o\left(\nu^{2}\right)}{\epsilon} \\
& \leq \frac{-\frac{3}{2} \mu^{2}-2 \epsilon R_{1}+2 \epsilon R_{2}+o\left(\nu^{2}\right)}{\epsilon} .
\end{aligned}
$$

In view of (86), (92) and the fact that $\left|R_{2}-R_{1}\right|=l+o(l)$, we have

$$
\mu^{2} \geq \frac{L l}{13 \epsilon}+o\left(\nu^{2}\right) \quad \text { and hence } \quad \mathcal{D}(u, \Psi) \leq-\frac{L l+o\left(\nu^{2}\right)}{14 \epsilon}
$$

Now consider two situations. If $o\left(\nu^{2}\right) \leq \frac{L l}{2}$, then $\mathcal{D}(u, \Psi) \leq-\frac{L l}{28 \epsilon}$. For $\Psi$, it is approximately equal to 0 in the segments $(A, B) \cup(C, D)$ and 1 in the segment $(B, C)$. So $\int_{A}^{D} \Psi^{2} \mathrm{~d} x=O(L+l+k)$. Again we have

$$
\partial^{2} \mathcal{E}(u, \Psi)=\mathcal{D}(u, \Psi)+\int_{A}^{D} \Psi^{2} \mathrm{~d} x=-\frac{L l}{28 \epsilon}+O(L+l+k)<0 !
$$

If $o\left(\nu^{2}\right) \geq \frac{L l}{2}$, then (88) holds and the same reasoning and test function (83) as in $\mathrm{LLS}_{1}$ can be used to conclude that this case is also unstable. 
Case of LLSL. Assume $R_{0} \geq-\frac{2 L}{3}$ and there is a long segment $(C, D)$ to the right of $(B, C)$. The configurations of $u$ and $u_{x}$ are illustrated in Figure 10.

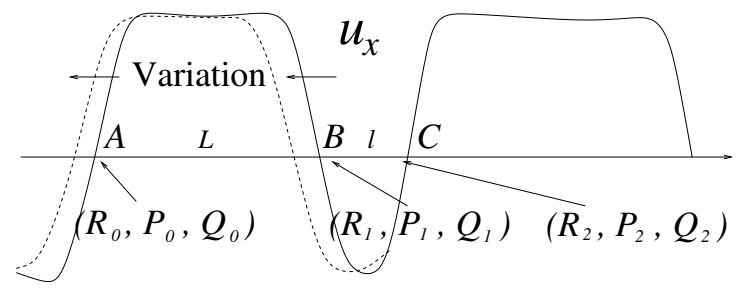

FIGURE 10. Variation of pattern LLSL.

In this case, at the point $C$, by (40), we have $P_{2}=Q_{2}+\epsilon R_{2}+O\left(\epsilon^{2}\right)$. In view of (89), (91) and (86), the following holds:

$$
o\left(\nu^{2}\right)+O\left(\epsilon^{2}\right)=\left(R_{1}-\frac{l}{2}+\epsilon\right) l+O\left(\epsilon^{2}\right) \geq \frac{L l}{13}+O\left(\epsilon^{2}\right)
$$

so that $\nu^{2} \geq o\left(\nu^{2}\right) \geq \frac{L l}{13}$. Using the same test function as in $L_{L S}$ also concludes that this pattern has a negative second variation.

Thus all the examples of the category LLS are unstable.

\subsubsection{Instability of $S L S$}

We refer to Figure 5. Let $\mu^{2}=e^{-\frac{m}{\epsilon}}$ and $\nu^{2}=e^{-\frac{l}{\epsilon}}$. First, the relations (55), (58) and (59) lead to $P_{1}^{\prime}=Q_{1}^{\prime}-\epsilon R_{2}+O\left(\epsilon^{2}\right)$ and $P_{2}=Q_{2}-2 Q_{2} \nu^{2}-\epsilon R_{2}+o\left(\nu^{2}\right)+O\left(\epsilon^{2}\right)$. Their comparison gives

$$
\begin{aligned}
P_{2} & =1-\nu^{2}-\epsilon R_{2}+o\left(\nu^{2}\right)+O\left(\epsilon^{2}\right) \\
Q_{2} & =1+\nu^{2}+o\left(\nu^{2}\right)+O\left(\epsilon^{2}\right) .
\end{aligned}
$$

Similarly

$$
P_{1}=1-\mu^{2}+\epsilon R_{1}+o\left(\mu^{2}\right)+O\left(\epsilon^{2}\right) .
$$

Using the map $\left(R_{2}, P_{2}, Q_{2}\right) \longrightarrow_{l}\left(R_{2}^{\prime}, P_{2}^{\prime}, Q_{2}^{\prime}\right)$, we have:

$$
\begin{aligned}
& P_{2}^{\prime}=1-\nu^{2}-\left(R_{2}-\frac{l}{2}+\epsilon\right) l+\epsilon R_{3}+o\left(\nu^{2}\right)+O\left(\epsilon^{2}\right) \\
& Q_{2}^{\prime}=Q_{2}-\left(R_{2}-\frac{l}{2}+\epsilon\right) l+O\left(\epsilon^{2}\right) .
\end{aligned}
$$

Without loss of generality, we can assume $R_{2}>\frac{L}{3}$. In addition, by the hypothesis of this case $-l \leq \frac{L}{2}$, we have the following estimate:

$$
\left(R_{2}-\frac{l}{2}+\epsilon\right) l \geq \frac{L l}{13}
$$

Now we divide this category into the following two cases each of which will be shown to have negative second variations. 
Case of SLSS. Assume that there is a short segment $(C, D)$ to the right of $(B, C)$. Figure 11 shows the configuration of $u_{x}$.

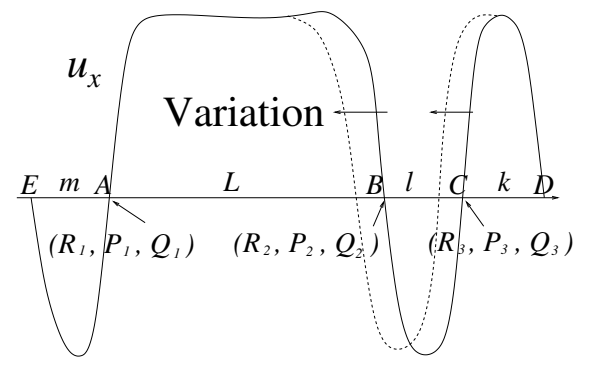

FiguRe 11. Variation of pattern SLSS - the first case.

Let $k=|C D| \leq 10 \epsilon|\ln \epsilon|$ and $\delta^{2}=e^{-\frac{k}{\epsilon}}$. Consider the point $C$. Using (50), we have:

$$
P_{3}=Q_{3}-2 Q_{3} \delta^{2}+\epsilon R_{3}+o\left(\epsilon \delta^{2}\right) .
$$

Since $P_{2}^{\prime}=P_{3}$ and $Q_{3}+Q_{2}^{\prime}=2,(99)$ gives:

$$
\begin{aligned}
& \delta^{2}=\left(R_{2}-\frac{l}{2}+\epsilon\right) l+o\left(\nu^{2}\right)+o\left(\delta^{2}\right)+O\left(\epsilon^{2}\right) \\
& P_{3}=1-\nu^{2}-\delta^{2}+\epsilon R_{3}+o\left(\nu^{2}\right)+o\left(\delta^{2}\right)+O\left(\epsilon^{2}\right) .
\end{aligned}
$$

Consider the following test function $\Psi$ which mimics the movement of interfaces at $B$ and $C$ :

Then $\mathcal{D}(u, \Psi)$ equals:

$$
\Psi_{x}= \begin{cases}-F_{x}\left(x-\frac{A+B}{2}, \frac{B-A}{2}\right) & x \in(A, B) \\ \left(\frac{1+\nu^{2}}{1-\nu^{2}}\right) G_{x}\left(x-\frac{B+C}{2}, \frac{C-B}{2}\right) & x \in(B, C) \\ \left(\frac{1+\delta^{2}}{1-\delta^{2}}\right) F_{x}\left(-x+\frac{C+D}{2}, \frac{D-C}{2}\right) & x \in(C, D) .\end{cases}
$$

$$
\begin{aligned}
\int_{A}^{D}\left(\epsilon^{2} \Psi_{x x}^{2}+\right. & \left.\Psi_{x}^{2}\right) \mathrm{d} x-2 \frac{\Psi_{x}^{2}(B)}{\left|u_{x x}(B)\right|}-2 \frac{\Psi_{x}^{2}(C)}{\left|u_{x x}(C)\right|} \\
= & \frac{1}{\epsilon}+\left(\frac{1+\nu^{2}}{1-\nu^{2}}\right)^{2} \frac{2\left(1-2 \nu^{2}\right)}{\epsilon}+\left(\frac{1+\delta^{2}}{1-\delta^{2}}\right)^{2} \frac{\left(1-4 \delta^{2}\right)}{\epsilon}-\frac{2}{\epsilon} \frac{1}{P_{2}}-\frac{2}{\epsilon} \frac{1}{P_{3}} \\
= & \frac{1}{\epsilon}+\frac{2+4 \nu^{2}}{\epsilon}+\frac{1+o\left(\delta^{2}\right)}{\epsilon} \\
& -\frac{2}{\epsilon} \frac{1}{1-\nu^{2}-\epsilon R_{2}+o\left(\nu^{2}\right)+O\left(\epsilon^{2}\right)}-\frac{2}{\epsilon} \frac{1}{1-\nu^{2}-\delta^{2}+\epsilon R_{3}++o\left(\nu^{2}\right)+o\left(\delta^{2}\right)+O\left(\epsilon^{2}\right)} \\
= & \frac{-2 \delta^{2}-2 \epsilon\left(R_{2}-R_{3}\right)+o\left(\nu^{2}\right)+o\left(\delta^{2}\right)}{\epsilon} .
\end{aligned}
$$

Hence, by (101), (102) and the fact that $\left|R_{2}-R_{3}\right|=l+o(l)$, we have

$$
\mathcal{D}(u, \Psi) \leq-\frac{L l+o\left(\nu^{2}\right)}{7 \epsilon} .
$$

Again, we consider two cases. If $o\left(\nu^{2}\right) \leq \frac{L l}{2}$, then

$$
\partial^{2} \mathcal{E}(u, \Phi)=-\frac{L l}{14 \epsilon}+l+o(l)<0 .
$$


If $o\left(\nu^{2}\right) \geq \frac{L l}{2}$, then we move the interfaces at $A$ and $B$ as shown in Figure 12:

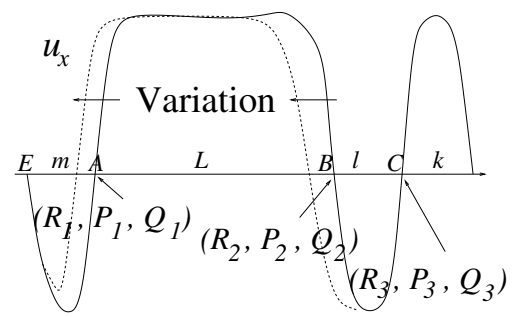

FIGURE 12. Variation of pattern SLSS - the second case.

The corresponding test function is:

$$
\Psi_{x}= \begin{cases}\left(\frac{1+\mu^{2}}{1-\mu^{2}}\right) F_{x}\left(x-\frac{E+A}{2}, \frac{A-E}{2}\right) & x \in(E, A) \\ G_{x}\left(x-\frac{A+B}{2}, \frac{B-A}{2}\right) & x \in(A, B) \\ -\left(\frac{1+\nu^{2}}{1-\nu^{2}}\right) F_{x}\left(-x+\frac{B+C}{2}, \frac{C-B}{2}\right) & x \in(B, C) .\end{cases}
$$

Similarly we have:

$$
\begin{aligned}
\mathcal{D}(u, \Psi) & =\int_{E}^{C}\left(\epsilon^{2} \Psi_{x}^{2}+\Psi_{x}^{2}\right) \mathrm{d} x-2 \frac{\Psi_{x}^{2}(A)}{\left|u_{x x}(A)\right|}-2 \frac{\Psi_{x}^{2}(B)}{\left|u_{x x}(B)\right|} \\
& =\left(\frac{1+\mu^{2}}{1-\mu^{2}}\right)^{2} \frac{1-4 \mu^{2}}{\epsilon}+\frac{2}{\epsilon}+\left(\frac{1+\nu^{2}}{1-\nu^{2}}\right)^{2} \frac{1-4 \nu^{2}}{\epsilon}-\frac{2}{\epsilon} \frac{1}{P_{1}}-\frac{2}{\epsilon} \frac{1}{P_{2}} \\
& =\frac{-2 \mu^{2}-2 \nu^{2}+2 \epsilon R_{1}-2 \epsilon R_{2}+o\left(\mu^{2}\right)+o\left(\nu^{2}\right)}{\epsilon} .
\end{aligned}
$$

The assumption on $o\left(\nu^{2}\right)$ now gives:

$$
\partial^{2} \mathcal{E}(u, \Phi)=-\frac{L l}{\epsilon}+L+o(L)<0 \text { ! }
$$

Case of SLSL. Assume that there is a long segment $(C, D)$ to the right of $(B, C)$. In this case, we have $P_{3}=Q_{3}+\epsilon R_{3}$. Combining the fact $Q_{2}^{\prime}+Q_{3}=2$ together with (99) and (100) gives

$$
o\left(\nu^{2}\right)+O\left(\epsilon^{2}\right)=\left(R_{2}-\frac{l}{2}+\epsilon\right) l \geq \frac{L l}{13}(\text { by }(101)) .
$$

Hence $\nu^{2} \geq o\left(\nu^{2}\right) \geq\left(R_{2}-\frac{l}{2}+\epsilon\right) l \geq \frac{L l}{13}$. and we can then use the same $\Psi$ as in (105) to deduce that the second variation is negative.

Hence, we have also shown that all the cases of SLS are unstable. Claim II is thus proved.

\subsection{Proof of Claim III}

In this section, we assume that $u$ is a solution of (9) with only long segments. We will prove that $l_{i}=l_{i+1}$ for all $i$ and hence $u$ is periodic by Proposition 3.3. The approach taken here resembles the use of implicit function theorem. We will exploit again the solution formula given by the propagation map defined in Section 3 . We use the same notation as in that section. 
Consider the boundary value problem as stated in Proposition 3.3. Given $Q, Q^{\prime}$, using (35), a solution similar to (36) is given as follows:

$$
\begin{array}{ll}
A=\frac{Q e^{-\Lambda l}-Q^{\prime}}{\epsilon^{2} \Lambda\left(\Lambda^{2}-\lambda^{2}\right)\left(e^{\Lambda l}-e^{-\Lambda l}\right)}, & B=\frac{Q e^{\Lambda l}-Q^{\prime}}{\epsilon^{2} \Lambda\left(\Lambda^{2}-\lambda^{2}\right)\left(e^{\Lambda l}-e^{-\Lambda l}\right)} \\
C=\frac{-Q e^{-\lambda l}+Q^{\prime}}{\epsilon^{2} \lambda\left(\Lambda^{2}-\lambda^{2}\right)\left(e^{\lambda l}-e^{-\lambda l}\right)}, & D=\frac{-Q e^{\lambda l}+Q^{\prime}}{\epsilon^{2} \lambda\left(\Lambda^{2}-\lambda^{2}\right)\left(e^{\lambda l}-e^{-\lambda l}\right)}
\end{array}
$$

Then we can express the solution as follows:

$$
\begin{gathered}
R(=u(0))=\frac{1}{\epsilon^{2}\left(\Lambda^{2}-\lambda^{2}\right)}\left\{\frac{Q\left(e^{\Lambda l}+e^{-\Lambda l}\right)-2 Q^{\prime}}{\Lambda\left(e^{\Lambda l}-e^{-\Lambda l}\right)}-\frac{Q\left(e^{\lambda l}-e^{-\lambda l}\right)-2 Q^{\prime}}{\lambda\left(e^{\lambda l}-e^{-\lambda l}\right)}\right\} \\
R^{\prime}(=u(l))=\frac{1}{\epsilon^{2}\left(\Lambda^{2}-\lambda^{2}\right)}\left\{\frac{2 Q-Q^{\prime}\left(e^{\Lambda l}+e^{-\Lambda l}\right)}{\Lambda\left(e^{\Lambda l}-e^{-\Lambda l}\right)}-\frac{2 Q-Q^{\prime}\left(e^{\lambda l}+e^{-\lambda l}\right)}{\lambda\left(e^{\lambda l}-e^{-\lambda l}\right)}\right\} \\
P\left(=\epsilon u_{x x}(0)\right)=\frac{1}{\epsilon\left(\Lambda^{2}-\lambda^{2}\right)}\left\{\frac{\Lambda\left[Q\left(e^{\Lambda l}+e^{-\Lambda l}\right)-2 Q^{\prime}\right]}{\left(e^{\Lambda l}-e^{-\Lambda l}\right)}-\frac{\lambda\left[Q\left(e^{\lambda l}-e^{-\lambda l}\right)-2 Q^{\prime}\right]}{\left(e^{\lambda l}-e^{-\lambda l}\right)}\right\} \\
P^{\prime}\left(=\epsilon u_{x x}(l)\right)=\frac{1}{\epsilon\left(\Lambda^{2}-\lambda^{2}\right)}\left\{-\frac{\Lambda\left[2 Q-Q^{\prime}\left(e^{\Lambda l}+e^{-\Lambda l}\right)\right]}{\left(e^{\Lambda l}-e^{-\Lambda l}\right)}+\frac{\lambda\left[2 Q-Q^{\prime}\left(e^{\lambda l}+e^{-\lambda l}\right)\right]}{\left(e^{\lambda l}-e^{-\lambda l}\right)}\right\} .
\end{gathered}
$$

Similar expressions hold if $u_{x}<0$ for $x \in(0, l)$ (by changing $Q$ and $Q^{\prime}$ to $-Q$ and $-Q^{\prime}$ ).

The above results are now applied to the solution $u$. Let $R_{i}^{ \pm}=u\left(c_{i}^{ \pm}\right), P_{i}^{ \pm}=\epsilon u_{x x}\left(c_{i}^{ \pm}\right), Q_{i}^{ \pm}=\epsilon^{2} u_{x x x}\left(c_{i}^{ \pm}\right)$ and $l_{i}=c_{i}-c_{i-1}$. Since $R_{i}^{+}=R_{i}^{-}$and $P_{i}^{+}=P_{i}^{-}$, we have, for $i=1,2, \ldots N-1$ :

$$
\begin{array}{r}
-\frac{2 Q_{i-1}^{+}-Q_{i}^{-}\left(e^{\Lambda l_{i}}+e^{-\Lambda l_{i}}\right)}{\Lambda\left(e^{\Lambda l_{i}}-e^{-\Lambda l_{i}}\right)}+\frac{2 Q_{i-1}^{+}-Q_{i}^{-}\left(e^{\lambda l_{i}}+e^{-\lambda l_{i}}\right)}{\lambda\left(e^{\lambda l_{i}}-e^{-\lambda l_{i}}\right)} \\
=\frac{Q_{i}^{+}\left(e^{\Lambda l_{i+1}}+e^{-\Lambda l_{i+1}}\right)-2 Q_{i+1}^{-}}{\Lambda\left(e^{\Lambda l_{i+1}}-e^{-\Lambda l_{i+1}}\right)}-\frac{Q_{i}^{+}\left(e^{\lambda l_{i+1}}+e^{-\lambda l_{i+1}}\right)-2 Q_{i+1}^{-}}{\lambda\left(e^{\lambda l_{i+1}}-e^{-\lambda l_{i+1}}\right)}
\end{array}
$$

and $-\frac{\Lambda\left[2 Q_{i-1}^{+}-Q_{i}^{-}\left(e^{\Lambda l_{i}}+e^{-\Lambda l_{i}}\right)\right]}{\left(e^{\Lambda l_{i}}-e^{-\Lambda l_{i}}\right)}+\frac{\lambda\left[2 Q_{i-1}^{+}-Q_{i}^{-}\left(e^{\lambda l_{i}}+e^{-\lambda l_{i}}\right)\right]}{\left(e^{\lambda l_{i}}-e^{-\lambda l_{i}}\right)}$

$$
=\frac{\Lambda\left[Q_{i}^{+}\left(e^{\Lambda l_{i+1}}+e^{-\Lambda l_{i+1}}\right)-2 Q_{i+1}^{-}\right]}{\left(e^{\Lambda l_{i+1}}-e^{-\Lambda l_{i+1}}\right)}-\frac{\lambda\left[Q_{i}^{+}\left(e^{\lambda l_{i+1}}+e^{-\lambda l_{i+1}}\right)-2 Q_{i+1}^{-}\right]}{\left(e^{\lambda l_{i+1}}-e^{-\lambda l_{i+1}}\right)} .
$$

For convenience, we introduce the following notations:

$$
\theta(l)=\left(1-e^{-2 \lambda l}\right), \quad \eta(l)=\frac{e^{-\Lambda l}}{1-e^{-2 \Lambda l}}, \quad E(l)=\frac{1+e^{-2 \Lambda l}}{1-e^{-2 \Lambda l}} \quad \text { and } \sigma=\frac{\lambda}{\Lambda}=\epsilon+O\left(\epsilon^{2}\right) .
$$

As $Q_{i}^{+}+Q_{i}^{-}=2$, we can write $Q_{i}^{ \pm}=1 \pm \delta_{i}$ for some number $\delta_{i}$. Then $(106)$ and $(107)$ become: $(i=1,2, \ldots N-1)$

$$
\begin{array}{r}
2 \theta\left(l_{i+1}\right) e^{-\lambda l_{i}} \delta_{i-1}+2 \theta\left(l_{i}\right) e^{-\lambda l_{i+1}} \delta_{i+1}+ \\
-2 \sigma \theta\left(l_{i}\right) \theta\left(l_{i+1}\right)\left[\eta\left(l_{i+1}\right)\left(1+\delta_{i-1}+\eta\left(l_{i+1}\right) \delta_{i+1}+\left(\frac{E\left(l_{i}\right)+E\left(l_{i+1}\right)}{2}\right)+\theta\left(l_{i}\right)\left(1+e^{-2 \lambda l_{i+1}}\right)\right] \delta_{i}\right] \\
=\theta\left(l_{i+1}\right)\left(1-e^{-\lambda l_{i}}\right)^{2}-\theta\left(l_{i}\right)\left(1-e^{-\lambda l_{i+1}}\right)^{2} \\
-\sigma \theta\left(l_{i}\right) \theta\left(l_{i+1}\right)\left[E\left(l_{i}\right)-2 \eta\left(l_{i}\right)-\left(E\left(l_{i+1}\right)-2 \eta\left(l_{i+1}\right)\right)\right]
\end{array}
$$


and

$$
\begin{gathered}
2 \theta\left(l_{i+1}\right) e^{-\lambda l_{i}} \delta_{i-1}+2 \theta\left(l_{i}\right) e^{-\lambda l_{i+1}} \delta_{i+1}+\left[\theta\left(l_{i+1}\right)\left(1+e^{-2 \lambda l_{i}}\right)+\theta\left(l_{i}\right)\left(1+e^{-2 \lambda l_{i+1}}\right)\right] \delta_{i} \\
-2 \frac{\theta\left(l_{i}\right) \theta\left(l_{i+1}\right)}{\sigma}\left[\eta\left(l_{i}\right) \delta_{i-1}+\eta\left(l_{i+1}\right) \delta_{i+1}+\left(\frac{E\left(l_{i}\right)+E\left(l_{i+1}\right)}{2}\right) \delta_{i}\right] \\
=\theta\left(l_{i+1}\right)\left(1-e^{-\lambda l_{i}}\right)^{2}-\theta\left(l_{i}\right)\left(1-e^{-\lambda l_{i+1}}\right)^{2} \\
-\frac{\theta\left(l_{i}\right) \theta\left(l_{i+1}\right)}{\sigma}\left[E\left(l_{i}\right)-2 \eta\left(l_{i}\right)-\left(E\left(l_{i+1}\right)-2 \eta\left(l_{i+1}\right)\right)\right] .
\end{gathered}
$$

Let $\mathrm{l}=\left(l_{1}, l_{2}, \ldots l_{N}\right)^{T}$. We now introduce the following vectors $\mathrm{a}, \mathrm{b}(\mathrm{l}), \mathrm{e}(\mathrm{l}) \in R^{N-1}$ and matrices $\mathrm{A}(\mathrm{l}), \mathrm{D}(\mathrm{l})$, $\Theta(l) \in R^{(N-1) \times(N-1)}$ : (In the following, $(\mathrm{M})_{i}$ refers to the $i$-th component of $\mathrm{M}$ and the subscript $i$ ranges from 1 to $N-1$.)

$$
\begin{aligned}
(\mathrm{a})_{i}= & \delta_{i} \\
(\mathrm{~b}(\mathrm{l}))_{i}= & \theta\left(l_{i+1}\right)\left(1-e^{-\lambda l_{i}}\right)^{2}-\theta\left(l_{i}\right)\left(1-e^{-\lambda l_{i+1}}\right)^{2}=\theta\left(l_{i}\right) \theta\left(l_{i+1}\right)\left(\frac{1-e^{-\lambda l_{i}}}{1+e^{-\lambda l_{i}}}-\frac{1-e^{-\lambda l_{i+1}}}{1+e^{-\lambda l_{i+1}}}\right) \\
(\mathrm{e}(\mathrm{l}))_{i}= & E\left(l_{i}\right)-2 \eta\left(l_{i}\right)-\left(E\left(l_{i+1}\right)-2 \eta\left(l_{i+1}\right)\right)=\left(\frac{1-e^{-\Lambda l_{i}}}{1+e^{-\Lambda l_{i}}}-\frac{1-e^{-\Lambda l_{i+1}}}{1+e^{-\Lambda l_{i+1}}}\right) \\
(\mathrm{A}(\mathrm{l}) \mathrm{a})_{i}= & 2 \theta\left(l_{i+1}\right) e^{-\lambda l_{i}} \delta_{i-1}+2 \theta\left(l_{i}\right) e^{-\lambda l_{i+1}} \delta_{i+1} \\
& +\left[\theta\left(l_{i+1}\right)\left(1+e^{-2 \lambda l_{i}}\right)+\theta\left(l_{i}\right)\left(1+e^{-2 \lambda l_{i+1}}\right)\right] \delta_{i} \\
(\mathrm{D}(\mathrm{l}) \mathrm{a})_{i}= & \eta\left(l_{i}\right) \delta_{i-1}+\eta\left(l_{i+1}\right) \delta_{i+1}+\left(\frac{E\left(l_{i}\right)+E\left(l_{i+1}\right)}{2}-1\right) \delta_{i} \\
\Theta(\mathrm{l})= & \text { the }(N-1) \times(N-1) \text { diagonal matrix with } \Theta(\mathrm{l})_{i i}=\theta\left(l_{i}\right) \theta\left(l_{i+1}\right) \\
\mathrm{I}= & \text { the }(N-1) \times(N-1) \text { identity matrix. }
\end{aligned}
$$

(In the above definitions of $\mathrm{A}(\mathrm{l})$ and $\mathrm{D}(\mathrm{l})$, we set $\delta_{0}=\delta_{N}=0$.) The expressions (108) and (109) can now be concisely written as

$$
\begin{aligned}
\mathrm{A}(\mathrm{l}) \mathrm{a}-2 \sigma \Theta(\mathrm{l})(\mathrm{I}+\mathrm{D}(\mathrm{l})) \mathrm{a} & =\mathrm{b}(\mathrm{l})-\sigma \Theta(\mathrm{l}) \mathrm{e}(\mathrm{l}) \\
\text { and } \mathrm{A}(\mathrm{l}) \mathrm{a}-2 \frac{1}{\sigma} \Theta(\mathrm{l})(\mathrm{I}+\mathrm{D}(\mathrm{l})) \mathrm{a} & =\mathrm{b}(\mathrm{l})-\frac{1}{\sigma} \Theta(\mathrm{l}) \mathrm{e}(\mathrm{l}) .
\end{aligned}
$$

The above leads to $2(\mathrm{I}+\mathrm{D}(\mathrm{l})) \mathrm{a}=\mathrm{e}(\mathrm{l})$ and $\mathrm{A}(\mathrm{l}) \mathrm{a}=\mathrm{b}(\mathrm{l})$. Hence

$$
\mathrm{b}(\mathrm{l})=\frac{\mathrm{A}(\mathrm{l})}{2}(\mathrm{I}+\mathrm{D}(\mathrm{l}))^{-1} \mathrm{e}(\mathrm{l}) \text { or equivalently } \Theta(\mathrm{l})^{-1} \mathrm{~b}(\mathrm{l})=\frac{\Theta(\mathrm{l})^{-1} \mathrm{~A}(\mathrm{l})}{2}(\mathrm{I}+\mathrm{D}(\mathrm{l}))^{-1} \mathrm{e}(\mathrm{l}) .
$$

Component-wise, the above is equal to, for $i=1,2, \ldots N-1$ :

$$
\frac{1-e^{-\lambda l_{i}}}{1+e^{-\lambda l_{i}}}-\frac{1-e^{-\lambda l_{i+1}}}{1+e^{-\lambda l_{i+1}}}=\frac{1}{\theta\left(l_{i}\right) \theta\left(l_{i+1}\right)}\left\{\sum_{k=1}^{N-1} \frac{1}{2}\left(\mathrm{~A}(\mathrm{l})(\mathrm{I}+\mathrm{D}(\mathrm{l}))^{-1}\right)_{i k}\left(\frac{1-e^{-\Lambda l_{k}}}{1+e^{-\Lambda l_{k}}}-\frac{1-e^{-\Lambda l_{k+1}}}{1+e^{-\Lambda l_{k+1}}}\right)\right\} .
$$

Consider the following function and its derivative

$$
f_{a}(l)=\frac{1-e^{-a l}}{1+e^{-a l}}, f_{a}^{\prime}(l)=\frac{2 a e^{-a l}}{\left(1+e^{-a l}\right)^{2}} .
$$


Hence there are numbers $\left\{\tilde{l}_{i}, \hat{l}_{i}\right\}_{i=1}^{N-1}$ such that

$$
\frac{2 \lambda e^{-\lambda \tilde{l}_{i}}}{\left(1+e^{-\lambda \tilde{l}_{i}}\right)^{2}}\left(l_{i}-l_{i+1}\right)=\frac{1}{\theta\left(l_{i}\right) \theta\left(l_{i+1}\right)}\left\{\sum_{k=1}^{N-1} \frac{1}{2}\left(\mathrm{~A}(\mathrm{l})(\mathrm{I}+\mathrm{D}(\mathrm{l}))^{-1}\right)_{i k}\left(\frac{2 \Lambda e^{-\Lambda \hat{l}_{k}}}{\left(1+e^{-\Lambda \hat{l}_{k}}\right)^{2}}\left(l_{k}-l_{k+1}\right)\right)\right\} .
$$

Note that $\frac{E\left(l_{i}\right)+E\left(l_{i+1}\right)}{2}-1=\frac{2 e^{-2 \Lambda l_{i}}}{1-e^{-2 \Lambda l_{i}}}+\frac{2 e^{-2 \Lambda l_{i+1}}}{1-e^{-2 \Lambda l_{i+1}}}$. Since $10 \epsilon|\ln \epsilon| \leq l_{i} \leq o(1)$, all the entries of $\mathrm{D}(\mathrm{l})$ are bounded by $O\left(\epsilon^{10}\right)$ which implies that the entries of $(\mathrm{I}+\mathrm{D}(\mathrm{l}))^{-1}$ can be bounded by some $O(1)$ constants. Furthermore, we have

$$
\frac{2 \lambda e^{-\lambda \tilde{l}_{i}}}{\left(1+e^{-\lambda \tilde{l}_{i}}\right)^{2}}=O(1), \quad \frac{2 \Lambda e^{-\Lambda \hat{l}_{k}}}{\left(1+e^{-\Lambda \hat{l}_{k}}\right)^{2}} \leq O\left(\epsilon^{9}\right) \quad \text { and } \quad \frac{1}{2} l_{i} \leq \theta\left(l_{i}\right) \leq 2 l_{i}
$$

so that the entries of $\Theta(\mathrm{l})^{-1} \mathrm{~A}(\mathrm{l})(\mathrm{I}+\mathrm{D}(\mathrm{l}))^{-1}$ can be bounded by $O\left(\max _{i}\left(l_{i}^{-1}\right)\right)$. Applying these estimates to $(110)$, we have $\sum_{i}\left|l_{i}-l_{i+1}\right|^{2} \leq o(1) \sum_{i}\left|l_{i}-l_{i+1}\right|^{2}$ which leads to $l_{i}=l_{i+1}$ for all $i$. Hence $\mathrm{e}(\mathrm{l})=0$, a $=0$ and $Q_{i}^{ \pm}=1$. By Proposition 3.3, $u$ equals $Q^{N}(x)$ given by (3). Claim III is thus proved.

\section{Proof of Theorem 1.3}

The theorem follows easily by the formulas and approach we have been using. Let $l=K \epsilon|\ln \epsilon|$ for some $K$, $N=\frac{1}{l}$ be an integer and $u=Q^{N}(x)$. Then,

$$
\nu^{2}=e^{-\frac{l}{\epsilon}}=\epsilon^{K} \quad \text { and } \quad u(B)=\frac{l}{2}+o(l)=O(\epsilon|\ln \epsilon|) .
$$

Now the graphs of $u$ and $u_{x}$ are shown in the following Figure 13.
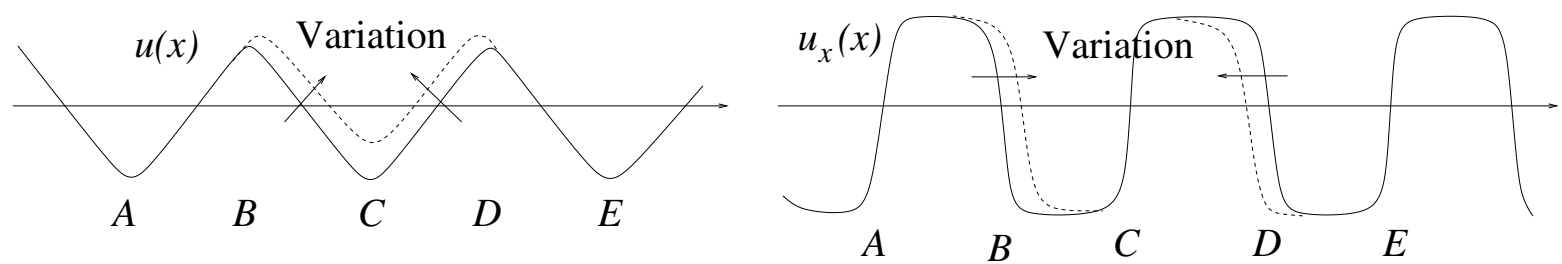

FIGURE 13. Variation of a periodic pattern with short period.

Consider the variation of $u_{x}$ given by:

$$
\Psi_{x}= \begin{cases}F_{x}\left(x-\frac{A+B}{2}, \frac{B-A}{2}\right), & x \in(A, B) \\ F_{x}\left(-x+\frac{B+C}{2}, \frac{C-B}{2}\right), & x \in(B, C) \\ -F_{x}\left(x-\frac{C+D}{2}, \frac{D-C}{2}\right), & x \in(C, D) \\ -F_{x}\left(-x+\frac{D+E}{2}, \frac{E-D}{2}\right), & x \in(D, E) .\end{cases}
$$


With the above, we have

$$
\begin{aligned}
\mathcal{D}(u, \Psi) & =\int_{A}^{E}\left(\epsilon^{2} \Psi_{x x}^{2}+\Psi_{x}^{2}\right) \mathrm{d} x-2 \frac{\Psi_{x}^{2}(B)}{\left|u_{x x}(B)\right|}-2 \frac{\Psi_{x}^{2}(D)}{\left|u_{x x}(D)\right|} \\
& =\frac{4}{\epsilon}\left(1-4 \nu^{2}+o\left(\nu^{4}\right)\right)-\frac{4}{\epsilon\left|u_{x x}(B)\right|} \frac{1}{\epsilon}\left(\frac{1-\nu^{2}}{1+\nu^{2}}\right)^{2} \\
& =\frac{4\left(1-4 \nu^{2}+o\left(\nu^{2}\right)\right)}{\epsilon}\left(1-\frac{1}{1-2 \nu^{2}-\epsilon u(B)+O\left(\epsilon^{2}\right)+o\left(\nu^{2}\right)}\right) \\
& \leq-\frac{7 \nu^{2}+O\left(\epsilon^{2}\right)}{\epsilon}
\end{aligned}
$$

where in the above, we have used (55) with $Q=\epsilon^{2} u_{x x x}(B)=1$ to express $\epsilon u_{x x}(B)$. Hence the overall second variation for the test function $\Psi$ is given by

$$
\partial^{2} \mathcal{E}(u, \Psi) \leq-\frac{7 \nu^{2}+O\left(\epsilon^{2}\right)}{\epsilon}+2 l+o(l)=\frac{-7 \epsilon^{K}+O\left(\epsilon^{2}|\ln \epsilon|\right)}{\epsilon}<0
$$

as long as $0<K<2$ and $\epsilon$ is small enough. Thus Theorem 1.3 is proved.

\section{A. Regularity properties of CRItical points of $\mathcal{E}$}

This section states some regularity results of the critical points of $\mathcal{E}$. They also relate the notion of stability introduced in Definitions 2.5 and 2.6 and the following definition of $H^{2}$-local-minimizers.

Definition A.1. A function $u \in \mathcal{V}$ is called a (strict) $H^{2}$-local minimizer of $\mathcal{E}$ if there is a $\delta>0$ such that

$$
\mathcal{E}(v)(>) \geq \mathcal{E}(u) \text { for all } v \in \mathcal{V} \text { with } 0<\|v-u\|_{H^{2}} \leq \delta .
$$

(In the literature, the above definition is frequently called a weak-local minimizer in contrast to strong-local minimizer in which the $H^{2}$-norm is replaced by the $L^{\infty}$-norm.)

Theorem A.2. Let $u \in \mathcal{V}$.

1. If $u$ is a stationary point, then the one-dimensional Lebesgue measure of the zeros of $u_{x}$ vanishes, i.e. $\mathcal{L}^{1}\left\{x: u_{x}(x)=0\right\}=0$. In addition, $u \in H^{3}([0,1])$ (and hence $\left.u_{x x} \in C^{\frac{1}{2}}([0,1])\right)$ and $\int_{0}^{1} u(x) \mathrm{d} x=0$. Let $V(x)=\int_{0}^{x} u(y) d y$. Then the following identities also hold:

$$
\begin{gathered}
\epsilon^{2} u_{x x x}-\left(\left|u_{x}\right|-1\right) \operatorname{sgn}\left(u_{x}\right)+V(x)=0, \text { a.e. } x \in[0,1] ; \\
\epsilon^{2} u_{x x}^{2}(x)-\left(\left|u_{x}\right|-1\right)^{2}+2 V(x) u_{x}(x)-u^{2}(x)=\epsilon^{2} u_{x x}(0)-1-u^{2}(0), \text { for all } x \in[0,1] .
\end{gathered}
$$

2. There exists a $\delta_{*}>0$ (independent of $\epsilon$ ) such that any stationary point $u$ with $\mathcal{E}(u)<\delta_{*}$ belongs to $\mathcal{Z}$ and hence is a solution of (9) in the sense of Definition 2.2. In particular, $u_{x x x}$ satisfies the conditions (13) and (14) at the sign-changing zeros $c_{i}$ of $u_{x}$ and hence $u_{x x}\left(c_{i}\right) \neq 0$ by the Proposition 2.3.

3. Any $H^{2}$-local minimizer $u$ is a stationary point. Hence if further $\mathcal{E}(u)<\delta_{*}$ (same as in the previous statement), then $u \in \mathcal{Z}$. In addition, $u_{x}(x) \neq 0$ for $x \neq c_{i}$, i.e. $u_{x}$ does not have any interior zeros.

4. If $u \in \mathcal{Z}$ is an $H^{2}$-local minimizer, then it is weakly-stable.

5. If $u \in \mathcal{Z}$ is a stable stationary point such that $u_{x}(x) \neq 0$ for $x \neq c_{i}$, i.e. $u_{x}$ does not have any interior zeros, then $u$ is a strict $H^{2}$-local minimizer.

The proof of the above theorem is elementary but somewhat technical and lengthy due to our choice of the non-smooth double-well potential $W(\cdot)$. For the sake of space, it is hence omitted but can be found in [18]. 


\section{B. Explicit examples}

In this section, we provide some simple explicit examples of unstable solutions of (9) to illustrate that the various cases considered in the proof of Theorem 1.2 can actually occur.

We will find $4 L$-periodic functions which solves the following unit cell problem (Fig. 14):

$$
u_{x}>0 \text { for } x \in(-l, l) \text {; }
$$

$u_{x}<0$ for $x \in(-L,-l) \bigcup(l, L)$;

and $u(x)=-u(-x)$ for $x \in(-L, L)$.

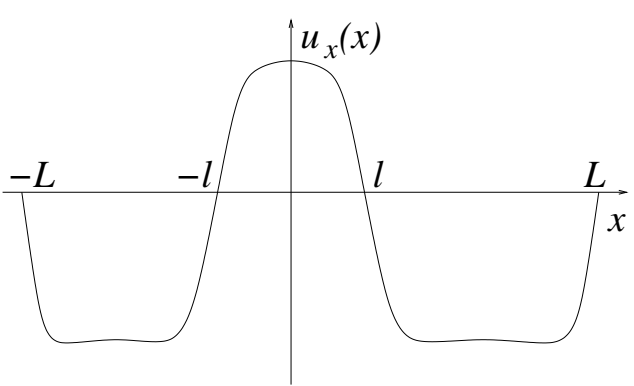

Figure 14. Explicit construction of an unstable pattern.

By symmetry and (35), it suffices to consider $u$ given by the form:

$$
u=\left\{\begin{array}{l}
A\left(e^{\Lambda x}-e^{-\Lambda x}\right)+B\left(e^{\lambda x}-e^{-\lambda x}\right), x \in(-l, l) \\
C e^{\Lambda x}+D e^{-\Lambda x}+E e^{\lambda x}+F e^{-\lambda x}, x \in(l, L) .
\end{array}\right.
$$

coinditidns for, there are seven unknowns $A, B \ldots F$ and $l$. They are found by the following boundary and jump

$$
\begin{gathered}
u_{x}\left(l^{-}\right)=0 ; \quad u_{x}\left(l^{+}\right)=0 ; \\
u\left(l^{-}\right)=u\left(l^{+}\right) ; \\
u_{x x}\left(l^{-}\right)=u_{x x}\left(l^{+}\right) ; \\
\epsilon^{2}\left(u_{x x x}\left(l^{+}\right)-u_{x x x}\left(l^{-}\right)\right)=2 ; \\
u_{x}(L)=0 ; \epsilon^{2} u_{x x x}(L)=1 .
\end{gathered}
$$

The above correspond to the following system of equations:

$$
\begin{gathered}
\Lambda A\left(e^{\Lambda l}+e^{-\Lambda l}\right)+\lambda B\left(e^{\lambda l}+e^{-\lambda l}\right)=0 \\
\Lambda\left(C e^{\Lambda l}-D e^{-\Lambda l}\right)+\lambda\left(E e^{\lambda l}-F e^{-\lambda l}\right)=0 \\
A\left(e^{\Lambda l}-e^{-\Lambda l}\right)+B\left(e^{\lambda l}-e^{-\lambda l}\right)=C e^{\Lambda l}+D e^{-\Lambda l}+E e^{\lambda l}+F e^{-\lambda l} \\
\Lambda^{2} A\left(e^{\Lambda l}-e^{-\Lambda l}\right)+\lambda^{2} B\left(e^{\lambda l}-e^{-\lambda l}\right)=\Lambda^{2}\left(C e^{\Lambda l}+D e^{-\Lambda l}\right)+\lambda^{2}\left(E e^{\lambda l}+F e^{-\lambda l}\right) \\
-\Lambda^{3} A\left(e^{\Lambda l}+e^{-\Lambda l}\right)-\lambda^{3} B\left(e^{\lambda l}+e^{-\lambda l}\right)+\Lambda^{3}\left(C e^{\Lambda l}-D e^{-\Lambda l}\right)+\lambda^{3}\left(E e^{\lambda l}-F e^{-\lambda l}\right)=\frac{2}{\epsilon^{2}} \\
\Lambda\left(C e^{\Lambda L}-D e^{-\Lambda L}\right)+\lambda\left(E e^{\lambda L}-F e^{-\lambda L}\right)=0 \\
\Lambda^{3}\left(C e^{\Lambda L}-D e^{-\Lambda L}\right)+\lambda^{3}\left(E e^{\lambda L}-F e^{-\lambda L}\right)=\frac{1}{\epsilon^{2}} .
\end{gathered}
$$


Let $\gamma=\frac{\lambda}{\Lambda}$. Using (113) - (117), we obtain:

$$
\begin{aligned}
& A=-\frac{\lambda}{\Lambda}\left(\frac{e^{\lambda l}+e^{-\lambda l}}{e^{\Lambda l}+e^{-\Lambda l}}\right) B \\
& C=-\frac{\lambda}{\Lambda}\left(\frac{e^{\lambda l}+e^{-\lambda l}}{e^{\Lambda l}+e^{-\Lambda l}}\right) B+\frac{e^{-\Lambda l}}{\epsilon^{2} \Lambda^{3}\left(1-\gamma^{2}\right)} \\
& D=\frac{\lambda}{\Lambda}\left(\frac{e^{\lambda l}+e^{-\lambda l}}{e^{\Lambda l}+e^{-\Lambda l}}\right) B-\frac{e^{\Lambda l}}{\epsilon^{2} \Lambda^{3}\left(1-\gamma^{2}\right)} \\
& E=B-\frac{e^{-\lambda l}}{\epsilon^{2} \Lambda^{3} \gamma\left(1-\gamma^{2}\right)} \\
& F=-B+\frac{e^{\lambda l}}{\epsilon^{2} \Lambda^{3} \gamma\left(1-\gamma^{2}\right)} .
\end{aligned}
$$

Substituting the above into (118) and (119) leads to the following two expressions for $B$ :

$$
B=\frac{e^{\lambda(L-l)}+e^{-\lambda(L-l)}-1}{\epsilon^{2} \Lambda^{3} \gamma\left(1-\gamma^{2}\right)\left(e^{\lambda L}+e^{-\lambda L}\right)} \text { and } B=\frac{\left(e^{\Lambda(L-l)}+e^{-\Lambda(L-l)}-1\right)\left(e^{\Lambda l}+e^{-\Lambda l}\right)}{\epsilon^{2} \Lambda^{3} \gamma\left(1-\gamma^{2}\right)\left(e^{\Lambda L}+e^{-\Lambda L}\right)\left(e^{\lambda l}+e^{-\lambda l}\right)}
$$

and hence the following relationship between $l$ and $L$ :

$$
\frac{\left(e^{\lambda(L-l)}+e^{-\lambda(L-l)}-1\right)\left(e^{\lambda l}+e^{-\lambda l}\right)}{e^{\lambda L}+e^{-\lambda L}}=\left(\frac{1+e^{-2 \Lambda l}}{1+e^{-2 \Lambda L}}\right)\left(1-e^{-\Lambda(L-l)}+e^{-2 \Lambda(L-l)}\right) .
$$

We will use the above to determine the value(s) of $l$.

To simplify the following computations, we will assume that $L=K \epsilon|\ln \epsilon|$ for some sufficiently large $K$. Note that (120) has the trivial solution $l=\frac{L}{3}$ which corresponds to the $\frac{4 L}{3}$-periodic solution. We look for two additional solutions. (By Claim III of the proof of Th. 1.2, these are the only three solutions.)

1. $l=h \epsilon|\ln \epsilon|$ for some $h<\frac{K}{3}$.

In this case, $e^{-2 \Lambda L}, e^{-\Lambda(L-l)}$ and $e^{-2 \Lambda(L-l)}$ are all transcendentally small and thus can be ignored. Then we have:

$$
\begin{aligned}
e^{-2 \Lambda l} & =\frac{\left(e^{\lambda(L-l)}+e^{-\lambda(L-l)}-1\right)\left(e^{\lambda l}+e^{-\lambda l}\right)}{e^{\lambda L}+e^{-\lambda L}}-1=\frac{e^{\lambda(L-2 l)}+e^{-\lambda(L-2 l)}-\left(e^{\lambda l}+e^{-\lambda l}\right)}{e^{\lambda L}+e^{-\lambda L}} \\
& =\frac{(\lambda(L-2 l))^{2}-(\lambda l)^{2}+O\left(L^{3}\right)}{e^{\lambda L}+e^{-\lambda L}} .
\end{aligned}
$$

The above has a solution for $l$ satisfying:

$$
e^{-2 \Lambda l}=O\left(L^{2}\right)
$$

which belong to the case of $\operatorname{LLS}_{1}$ - see (82). The graph of $u$ is shown in Figure 15 .

2. $y=L-l=h \epsilon|\ln \epsilon|$ for some $h<\frac{K}{3}$.

Let $y=L-l$. By ignoring all the transcendentally small terms again, we obtain:

$$
\begin{aligned}
-e^{-\Lambda y}+e^{-2 \Lambda y} & =\frac{e^{\lambda(L-2 y)}+e^{-\lambda(L-2 y)}-\left(e^{\lambda(L-y)}+e^{-\lambda(L-y)}\right)}{e^{\lambda L}+e^{-\lambda L}} \\
& =-\frac{2 \lambda^{2} L y+o(L y)}{e^{\lambda L}+e^{-\lambda L}}
\end{aligned}
$$


which has a solution for $y$ such that

$$
e^{-\Lambda y}=O(L y)
$$

This belongs to the case of SLSS - see (101). The graph of $u$ is shown in Figure 16 .

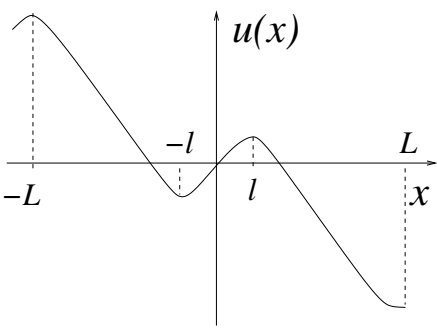

Figure 15. An LLS 1 unstable pattern.

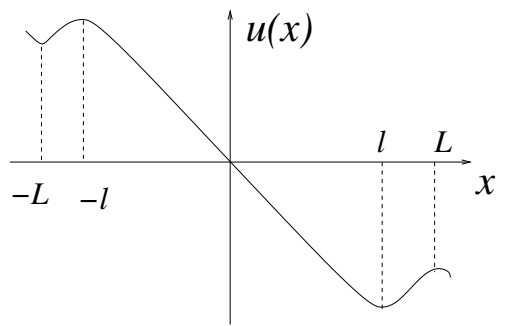

Figure 16. An SLSS unstable pattern.

By using the above approach together with some perturbation argument, it seems possible to construct other non-periodic unstable solutions. It is certainly interesting to characterize the structure of all critical points.

Acknowledgements. The author would like to thank Stefan Müller for introducing and patiently explaining this interesting problem to him. The hospitality of the Max Planck Institute at Leipzig, Germany is highly appreciated. The communication with Matthias Winter is also enjoyable and helpful. In addition, the comment from the referee substantially improves the first version of the paper. The author is partially supported by an NSF Grant.

\section{REFERENCES}

[1] R.A. Abeyaratne, C. Chu and R.D. James, Kinetics of materials with wiggly energies: theory and application to the evolution of twinning microstructures in a Cu-Al-Ni shape. Philos. Mag. Ser. A 73 (1996) $457-497$.

[2] G. Alberti and S. Müller, A New Approach to Variational Problems with Multiple Scales. Comm. Pure. Appl. Math. 54 (2001) 761-825.

[3] J. Ball and R.D. James, Fine phase mixtures as minimizers of the energy. Arch. Rat. Mech. Anal. 100 (1987) 13-52.

[4] J. Ball, R.D. James, Proposed experimental tests of a theory of fine structures and the two-well problem. Philos. Trans. R. Soc. Lond. A 338 (1992) 389-450.

[5] P.W. Bates and J. Xun, Metastable Patterns for the Cahn-Hilliard Equations, Part I. J. Diff. Eq. 111 (1994) $421-457$.

[6] J. Carr, M.E. Gurtin and M. Slemrod, Structured Phase Transitions on a Finite Interval. Arch. Rat. Mech. Anal. 86 (1984) 317-351.

[7] J. Carr and R.L. Pego, Metastable Patterns in Solutions of $u_{t}=\epsilon^{2} u_{x x}-f(u)$. Comm. Pure Appl. Math. 42 (1989) $523-576$.

[8] A.G. Khachaturyan, Theory of Structural Transformations in Solids. New York, Wiley-Interscience (1983).

[9] R.V. Kohn and S. Müller, Branching of twins near a austenite/twinned-martensite interface. Philos. Mag. Ser. A 66 (1992) 697-715.

[10] R.V. Kohn and S. Müller, Surface energy and microstructure in coherent phase transitions. Comm. Pure Appl. Math. 47 (1994) 405-435.

[11] R.V. Kohn and P. Sternberg, Local minimizers and singular perturbations. Proc. Roy. Soc. Edinburgh Sect. A 111 (1989) 69-84.

[12] S. Müller, Singular perturbations as a selection criterion for periodic minimizing sequences. Calc. Var. 1 (1993) 169-204.

[13] X. Ren, L. Truskinovsky, Finite Scale Microstructures in Nonlocal Elasticity. J. Elasticity 59 (2000) $319-355$.

[14] X. Ren and J. Wei, On the multiplicity of solutions of two nonlocal variational problems. SIAM J. Math. Anal. 31 (2000) 909-924.

[15] X. Ren and J. Wei, On energy minimizers of the diblock copolymer problem. Interfaces Free Bound. 5 (2003) $193-238$.

[16] L. Truskinovsky and G. Zanzotto, Ericksen's Bar Revisited: Energy Wiggles. J. Mech. Phys. Solids 44 (1996) $1371-1408$.

[17] A. Vainchtein, T. Healey, P. Rosakis and L. Truskinovsky, The role of the spinodal region in one-dimensional martensitic phase transitions. Physica D 115 (1998) 29-48.

[18] N.K. Yip, manuscript (2005). 